OPEN ACCESS

Edited by:

Olivier Cuvillier,

UPR8241 Laboratoire de Chimie de Coordination (LCC), France

Reviewed by:

Yuyan Han,

University of Northern Colorado,

United States

Sabina EP,

VIT University, India

*Correspondence:

Jing $L i$

100392@cqmu.edu.cn

Specialty section:

This article was submitted to

Pharmacology of Anti-Cancer Drugs,

a section of the journal

Frontiers in Oncology

Received: 04 June 2021

Accepted: 29 July 2021

Published: 16 August 2021

Citation:

Zeng D, Wang Y, Chen Y, Li D,

Li G, Xiao H, Hou J, Wang Z, Hu L, Wang L and Li J (2021) Angelica Polysaccharide Antagonizes 5-FU-

Induced Oxidative Stress Injury

to Reduce Apoptosis in the

Liver Through Nrf2 Pathway.

Front. Oncol. 11:720620.

doi: 10.3389/fonc.2021.720620

\section{Angelica Polysaccharide Antagonizes 5-FU-Induced Oxidative Stress Injury to Reduce Apoptosis in the Liver Through Nrf2 Pathway}

\author{
Di Zeng ${ }^{1}$, Yaping Wang ${ }^{1}$, Yi Chen ${ }^{1}$, Danyang $\mathrm{Li}^{2}$, Guoli $\mathrm{Li}^{1}$, Hanxianzhi Xiao ${ }^{1}$, Jiyin Hou ${ }^{1}$, \\ Ziling Wang ${ }^{1}$, Ling $\mathrm{Hu}^{1}{ }^{1}$, Lu Wang ${ }^{1}$ and Jing $\mathrm{Li}^{1{ }^{*}}$ \\ ${ }^{1}$ Laboratory of Stem Cells and Tissue Engineering, Department of Histology and Embryology, Chongqing Medical University, \\ Chongqing, China, ${ }^{2}$ Centre for Lipid Research \& Key Laboratory of Molecular Biology for Infectious Diseases, Chongqing \\ Medical University, Chongqing, China
}

Oxidative stress induced by chemotherapeutic agents causes hepatotoxicity. 5Fluorouracil (5-FU) has been found to have a variety of side effects, but its toxic effect on the liver and the mechanism are still unclear. Angelica polysaccharide (ASP), the main active ingredient of Dang Gui, has antioxidative stress effects. In this study, we investigated the antagonistic effects of ASP on 5-FU-induced injury in the mouse liver and human normal liver cell line MIHA and the possible mechanism. Our results show that ASP inhibited 5-FU-induced the decrease in Bcl-2 protein and the increase in Bax protein. ASP alleviated 5-FU-induced the increase in alanine aminotransferase (ALT), triglyceride (TG), and aspartate aminotransferase (AST) content; hepatic steatosis; and liver fibrosis. ASP restored 5-FU-induced swelling of mitochondria and the endoplasmic reticulum. 5FU promoted the expression of Keap1 and increased the binding to NF-E2-related factor 2 (Nrf2) to reduce the nuclear translocation of Nrf2, thereby weakening the transcriptional activity of Nrf2 to inhibit the expression of $\mathrm{HO}-1$; reducing the activity of $\mathrm{GSH}, \mathrm{SOD}$, and CAT to increase ROS content; and aggravating DNA damage (indicated by the increase in 8-OHdG). However, ASP reversed these reactions. In conclusion, ASP attenuated the 5FU-induced Nrf2 pathway barrier to reduce oxidative stress injury and thereby inhibit the disorder of lipid anabolism and apoptosis. The study provides a new protectant for reducing the hepatic toxicity caused by 5-FU and a novel target for treating the liver injury.

Keywords: 5-fluorouracil, ASP, oxidative stress, Nrf2, hepatotoxicity, apoptosis 


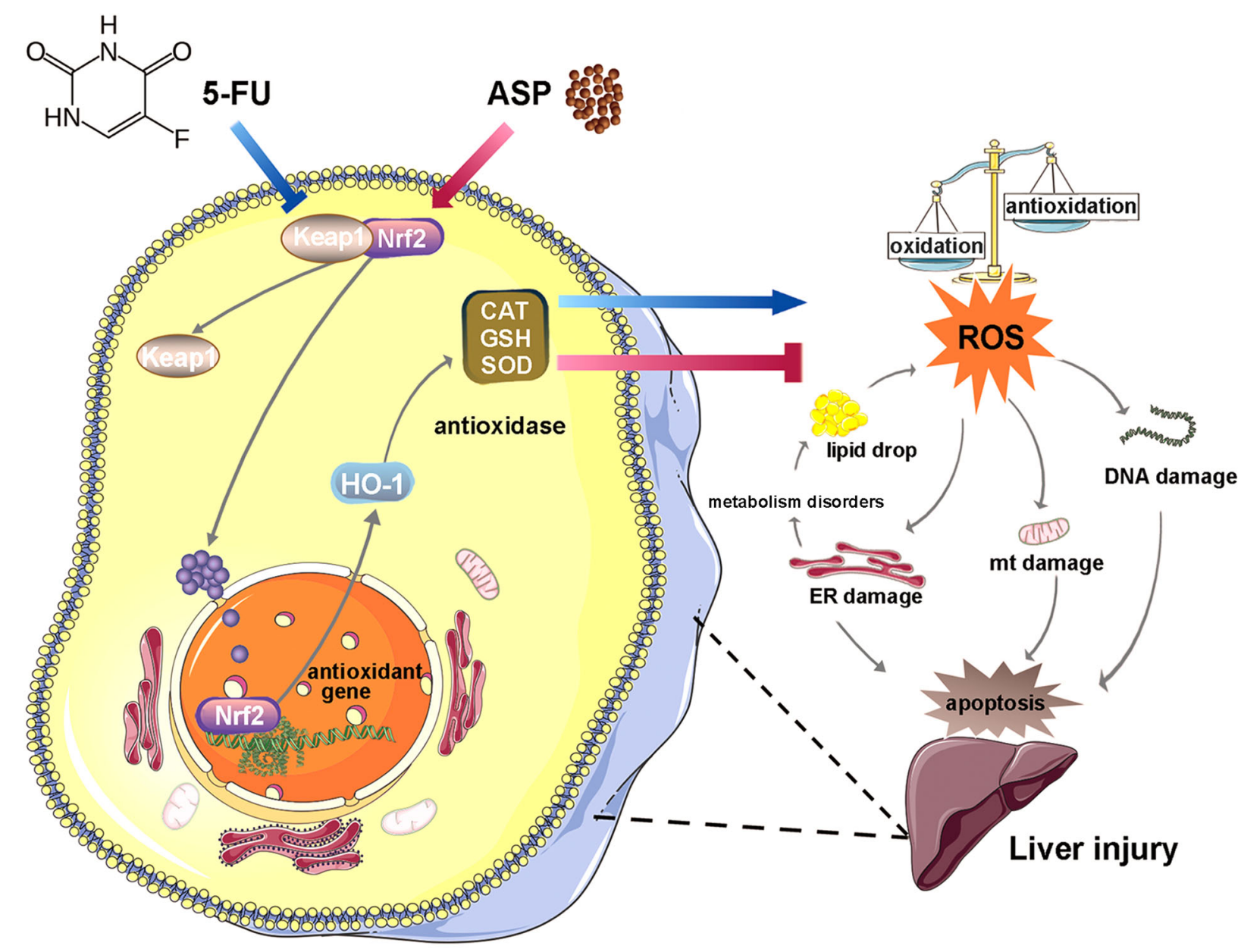

GRAPHICAL ABSTRACT

\section{INTRODUCTION}

5-Fluorouracil (5-FU) is a cytotoxic chemotherapy medication used to treat cancer, such as colorectal cancer, oesophageal cancer and stomach cancer, with the effect of blocking thymidylate synthase $(1,2)$. Administration of 5-FU results in a deficiency of deoxythymidine monophosphate, so that rapidly dividing cancer cells undergo cell death by thymineless death (3). The typical adverse effects of 5-FU are nausea, vomiting, mucositis, and diarrhoea (4). In addition, 5-FU can promote the production of free radicals and cause mitochondrial damage, leading to cardiotoxicity (5). Also, oxidative stress is vital in mediating 5-FU-induced renal injury (6). However, there are very few reports on the liver toxicity caused by $5-\mathrm{FU}$. A study has shown that after intraperitoneal injection of 5-FU, there are pathological and ultrastructural abnormalities of liver tissue (7), but the possible mechanism has not been elucidated.

Factors such as autoimmune attacks of liver cells, viral infections and drug abuse can lead to liver injury (8). Liver injury is regulated by multiple factors, and the mechanism of its pathogenesis and development has not been fully elucidated. Reactive oxygen species (ROS) are potentially vital in cell signaling and disease. The excessive deposition of free fatty acids and triglycerides (TGs) will lead to liver lipid metabolism disorders, which increase the liver's susceptibility to oxidative stress (OS), resulting in a large amount of ROS, which leads to apoptosis (9). Additionally, some antioxidants have been shown to improve lipotoxicity (10). A study found that inhibiting the inflammatory apoptotic response and the production of ROS could improve liver apoptosis in mice $(11,12)$. In addition, inhibiting the production of liver ROS could ameliorate liver fibrosis (13). Therefore, reducing the production of ROS to reduce liver injury is very important.

Various biological processes of the liver involve the Nrf2 antioxidant system, such as regulating the metabolism of liver substances, detoxification, and regeneration of liver cells, which are potential targets for treating liver diseases and liver injury (14). Nrf2 is a crucial transcription factor with an antioxidant effect (15). Under normal conditions, keap1 and Nrf2 exist as dimers in the cytoplasm. Once exposed to oxidative stress, the 
two dissociate and $\mathrm{Nrf} 2$ is transcribed to regulate the transcriptional activation of a series of cytoprotective genes (16). HO-1 is one of the antioxidant response kinases downstream of Nrf2. It has the ability to reduce liver damage (17). Studies suggest that the activation of Nrf2 reduces liver injury (18-20). There has been an increase in interest in finding potential protective agents against adverse reactions associated with chemotherapy. Adjuvant therapy with drugs with antioxidant function and minor side effects may be an effective measure to reduce the hepatotoxicity caused by 5 -FU.

Angelica polysaccharide (ASP) is the main active component in Dang Gui with the functions of antioxidation, immune regulation, and radiation protection (21-24). Our previous study showed that ASP slows down hematopoietic stem cells senescence by reducing oxidative stress (25); our other study showed that ASP could also antagonize the inhibitory effect of 5FU on bone marrow stromal cells (2). The damage to some organs induced by $5-\mathrm{FU}$ is closely related to oxidative stress, so we hypothesize that 5-FU could aggravate oxidative stress and lead to liver injury, while ASP could activate the antioxidant response, promote ROS clearance, and reduce lipid deposition and cell apoptosis to prevent 5-FU-induced liver injury.

\section{MATERIALS AND METHODS}

\section{Reagents and Antibodies}

5-FU (F6627-1G, purity $\geq 99 \%$ ) was purchased from SigmaAldrich (Saint Louis, Missouri, USA) and dissolved in dimethyl sulfoxide and phosphate-buffered saline (PBS). Angelica polysaccharide was purchased from Shanxi Ciyuan Biotechnology Co. Ltd. (Xi'an, China) with a purity of $\geq 95 \%$, dissolved in normal saline (NS). The primary antibody used are as follows: HO-1 (Abcam, cat. no. ab68477, Cambridge, UK), Nrf2 (CST, cat. no. D1Z9C, Danvers, MA, USA), Keap1 (CST, cat. no. D6B12), $\beta$-actin (CST, cat. no.13E5), Bcl-2 (Santa Cruz, cat. no.7382, CA, USA), Bax (Santa Cruz, cat. no.20067), 8OHdG (Bioss, cat. no.1278R, Beijing, China), and Lamin B1 (Proteintech, cat. no. 66095, PA, USA).

\section{Animals and Treatments}

C57BL/6J mice (male, 6-8 weeks old) were obtained from the Laboratory Animal Center of Chongqing Medical University $(n=60)$. The mice were randomly divided into four groups: the control group ( $n=15)$, ASP group $(n=15), 5$-FU group $(n=15)$, and 5 -FU + ASP group $(n=15)$. In the 5 -FU group, 5 -FU (150 $\mathrm{mg} / \mathrm{kg}$ ) was injected intraperitoneally into mice, and after $6 \mathrm{~h}$, normal saline $(10 \mathrm{~mL} / \mathrm{kg} / \mathrm{d})$ was injected for $7 \mathrm{~d}$. In the $5-\mathrm{FU}+$ ASP group, after $6 \mathrm{~h}$ of 5 -FU injection, ASP (100 mg/kg/day) was injected intraperitoneally daily for $7 \mathrm{~d}$. In the ASP group, ASP (100 mg/kg/day) was injected for $7 \mathrm{~d}$. All control animals were given $10 \mathrm{~mL} / \mathrm{kg} / \mathrm{d}$ normal saline intraperitoneally for $7 \mathrm{~d}$. All animal experiments were approved by the Chongqing Medical University Animal Care and Use Committee and performed following institutional and national guidelines.

\section{Cell Culture and Culture Condition}

The human normal hepatic MIHA cell line was a gift from the Centre for Lipid Research \& Key Laboratory of Molecular Biology for Infectious Diseases (Chongqing, China). Cells were cultured in DMEM medium supplemented with $10 \%$ fetal bovine serum and $100 \mu \mathrm{g} / \mathrm{mL}$ penicillin and streptomycin and incubated at $37^{\circ} \mathrm{C}$ in $5 \% \mathrm{CO}_{2}$ as described (26).

\section{Biochemical Analysis}

After the mice were sacrificed, an appropriate amount of tissue samples was taken, added to precooled PBS, homogenized in an ice bath, and centrifuged for $10 \mathrm{~min}$ at $4^{\circ} \mathrm{C}$. The BCA kit (Beyotime Institute of Biotechnology, Shanghai, China) was used to detect the protein concentration in the supernatant. After that, the activity of glutathione (GSH), catalase (CAT), and superoxide dismutase (SOD), and the contents of alanine aminotransferase (ALT), aspartate aminotransferase (AST), total cholesterol (TC), triglyceride (TG), malondialdehyde (MDA), and nitric oxide (NO) were determined with the corresponding biochemical kits (Jian Cheng Biotechnology, Nanjing, China) as previously described by our laboratory (27). All procedures were performed based on the manufacturer's protocols (28).

\section{Measurement of Reactive Oxygen Species}

ROS content was detected by a DCFH-DA probe (SigmaAldrich, MO, USA). After incubation with ROS staining solution for $30 \mathrm{~min}$ at $37^{\circ} \mathrm{C}$, MIHA cells or liver sections were washed three times with PBS. The fluorescence was detected by a fluorescence microplate reader for cells or microscope for liver sections (Olympus, Tokyo, Japan).

\section{Ultrastructure of the Mice Liver}

$1 \mathrm{~mm}^{3}$ of the fresh liver was immediately immobilized in sufficient glutaraldehyde. Then dehydrated with graded ethanol and embedded within the epoxy resin. After polymerization, 70 $\mathrm{nm}$ ultrathin sections were cut and then stained with uranyl acetate and lead citrate as described (28). The cells were observed by transmission electron microscopy (TEM).

\section{Histopathological Staining}

Paraffin sections of liver tissue were prepared with a thickness of $5 \mu \mathrm{m}$. Tissue sections were stained with hematoxylin-eosin (HE) to assess the general liver structure as previously described by our laboratory (28). Cells were also stained with a Masson kit (Solabio, Beijing, China) to measure changes in collagen fibers. Images were obtained using microscopy (Olympus, Tokyo, Japan).

\section{TUNEL Assay}

Paraffin sections of liver tissue were prepared, and the InSitu Cell DeathDetection Kit (Roche) was used to conduct experiments according to the instructions. The nuclei of apoptotic cells were dark brown. The sections were stained lightly with hematoxylin as described (29). Images were obtained using microscopy (Olympus, Tokyo, Japan). 


\section{Oil Red O Staining}

The frozen liver sections of $8 \mu \mathrm{m}$ thick, and cells were stained with Oil Red O (Sigma, USA) for $10 \mathrm{~min}$, washed off the dye solution, and stained with hematoxylin for $1 \mathrm{~min}$. The slides were fixed with glycerol and phosphate buffered saline (glycerol: PBS = 1:1) and observed under a microscope (Olympus, Tokyo, Japan).

\section{Immunohistochemistry Staining}

The immunohistochemistry SP9000 kit (Zhongshan Chemical Industry, Beijing, China) was used for the experiment. The primary antibody against Keap1 was added and incubated at $4^{\circ} \mathrm{C}$ overnight. The sections were reheated at $37^{\circ} \mathrm{C}$ for $30 \mathrm{~min}$. The secondary antibody was added and incubated for $30 \mathrm{~min}$. Incubated with streptavidin-HRP at room temperature for $30 \mathrm{~min}$ and then incubated with diaminobenzidine stained substrate and stained with Mayer's hematoxylin, as we did before (26). Keap1 proteins were represented as brown or yellow granular clumps. Images were obtained using microscopy (Olympus, Tokyo, Japan).

\section{Reverse Transcription-Quantitative PCR Assay}

RT-qPCR assay was performed in a Real-Time PCR Detection System (Bio-Rad Laboratories, Inc.) according to the procedures provided by the reagent provider (Bio-Rad Laboratories, Inc.) as we described (26). The relative quantification values for each gene were calculated by the $2^{-\Delta \Delta \mathrm{Ct}}$ method using $\beta$-actin as an internal reference. The primers used (Sangon Biotech Co., Ltd. Shanghai, China) are shown in Supplementary Table 1.

\section{Immunoblot Assay}

The protease inhibitor (TargetMol, USA) and Reagents kit (Thermo Fisher, USA) were used to extract nuclear and cytosolic protein fractions. The proteins were performed to SDSPAGE. Next, the separated proteins were transferred to the polyvinylidene difluoride (PVDF) membrane. After blocking the PVDF membranes with $5 \%$ nonfat milk for $1 \mathrm{~h}$, the primary antibodies against Bcl-2, Bax, Nrf2, Keap1 and HO-1 were added and incubated at $4^{\circ} \mathrm{C}$ overnight. And added the corresponding secondary antibody to the membranes and incubated for $1 \mathrm{~h}$ at room temperature. The immunoblots were analyzed using the ChemiDoc Touch Imaging System (Bio-Rad Laboratories, Inc.) as we described (26). The Image Lab 5.2.1 (BIO-RAD, Hercules, CA, USA) was used to analyze the intensities of the protein bands.

\section{Immunofluorescence Staining}

Frozen sections of liver tissue and cells were fixed with paraformaldehyde. And the cells were permeabilized with $1 \%$ goat serum albumin blocking solution containing $0.5 \%$ Triton $\mathrm{X}$ 100 for 30 minutes. Then, primary antibodies against 8-OHdG, $\mathrm{Nrf} 2$, and $\mathrm{HO}-1$ were added and incubated at $4^{\circ} \mathrm{C}$ overnight. Next, the sections and MIHA cells were stained with the secondary antibody for $1 \mathrm{~h}$ at $37^{\circ} \mathrm{C}$. DAPI was used to stain the nuclei for 5 minutes (26). Images were captured with fluorescence microscopy (Olympus, Tokyo, Japan).

\section{Statistical Analysis}

The data in the text are presented as the mean \pm SD (standard deviation). Statistical analysis was performed with GraphPad Prism 7.0 software (San Diego, CA, USA). One-way ANOVA was used to determine the differences when there were four groups. Student's t-test was used to evaluate the difference between when there were two groups. $p<0.05$ was considered as statistically significant.

\section{RESULTS}

\section{ASP Alleviated 5-FU-Induced Histopathology and Apoptosis in the Liver of Mice}

The liver appearance of the mice was yellow-tinged and greasy in the 5-FU treatment group, while the livers of the other three groups were pink-red (Figure 1A). Additionally, the liver weight was decreased in the 5-FU group; however, ASP treatment restored the liver weight decrease induced by 5 -FU (Figure 1B). Due to the weight loss of mice in the 5-FU group, even though the liver weight was reduced, the difference in the liver index between the groups was not statistically significant (Figure 1C). We then questioned whether treatment with ASP might alleviate liver damage. The results showed that ASP treatment alleviated the 5-FU-induced the increase of ALT and AST in the serum and liver (Figures 1D-G). Histological examination showed that hepatic lobules structure damage and vacuolization of hepatocytes in the 5-FU group (Figures 1H, I). Further analysis of the process of apoptosis showed that the count of TUNEL-positive cells in 5-FU-treated mice also decreased after ASP treatment (Figures 1J, K). The results indicated that the level of $\mathrm{Bcl}-2$ decreased in the 5-FU treatment group, while the level of Bax was increased, and ASP treatment reversed these changes. 5-FU-induced hepatocyte apoptosis was inhibited after ASP treatment (Figures 1L-N). These results indicated that ASP effectively reduced the structural damage to liver tissue and liver apoptosis caused by 5 -FU.

\section{ASP Attenuated 5-FU-Induced Liver Lipid Deposition In Vivo}

The biochemical indexes of liver tissue showed that the concentration of TG in mice liver in the 5-FU group was increased, and ASP treatment alleviated the abnormal increase in TG (Figure 2B). However, there was no significant difference in TC levels between the 5-FU group and the control group (Figure 2A). Oil Red $\mathrm{O}$ staining demonstrated more lipid deposition in the 5-FU group, whereas ASP treatment alleviated this abnormal phenomenon (Figures 2C,D). Masson staining showed the increasing area of fibrosis in the portal site of the mice liver in the 5-FU group, but ASP reduced this increased area of fibrosis (Figures 2E, F). Additionally, changes in liver ultrastructure were observed by electron 
A

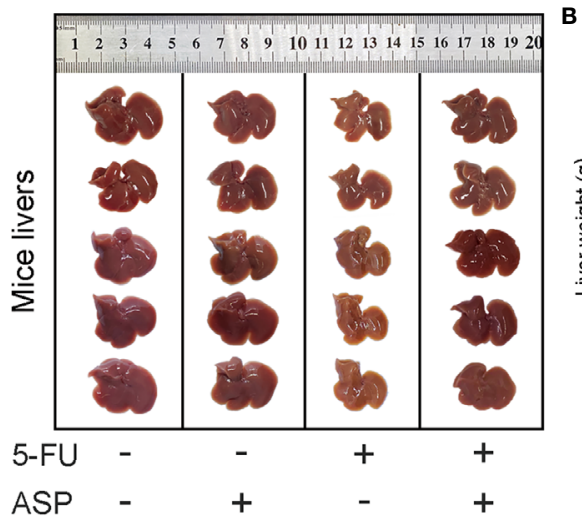

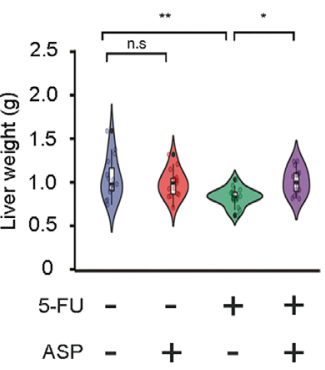

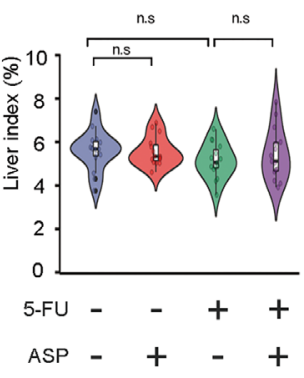

D

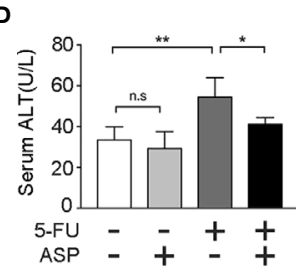

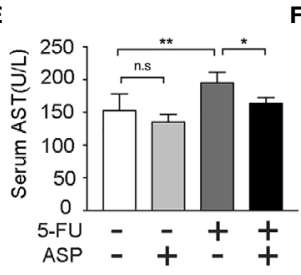

F

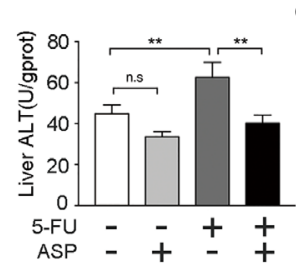

G

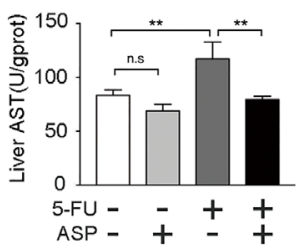

H

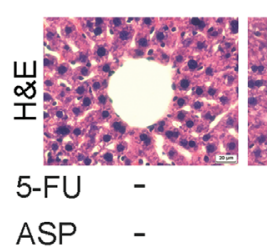

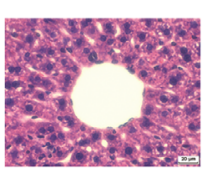

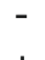

$+$

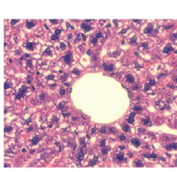

$+$

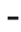

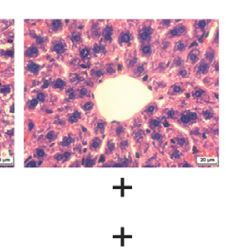

I

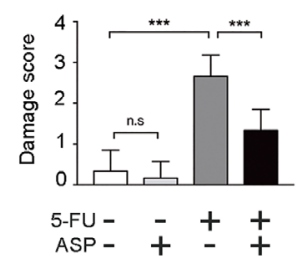

J
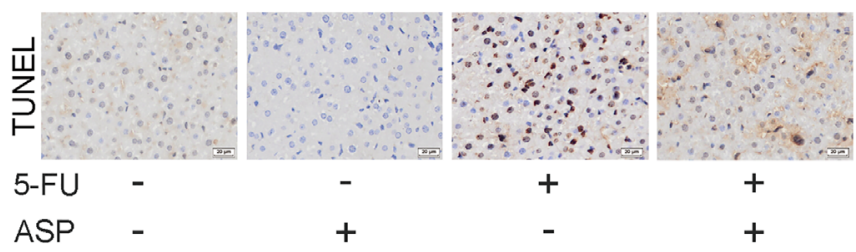

K

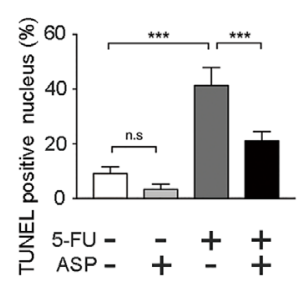

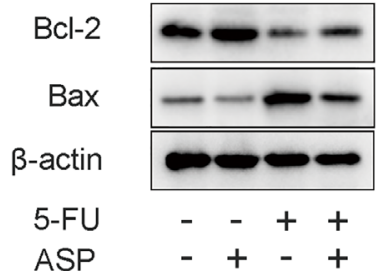

M

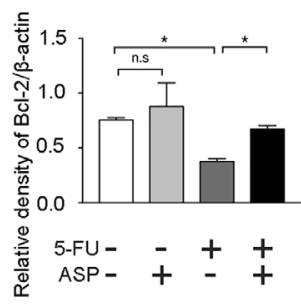

N

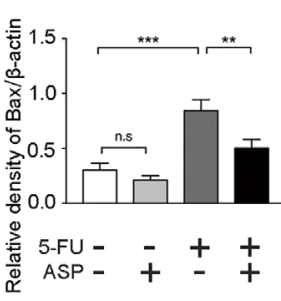

FIGURE 1 | ASP attenuated 5-FU-induced liver histopathological damage and apoptosis in mice. (A) The appearance of the livers of each group was examined. (B) The weight of livers of each group was examined ( $n=15 /$ group). (C) The liver index was calculated by the ratio of the liver weight (g) to the mouse body weight (g) $\times 100$ ( $n=15 /$ group). (D, E) The levels of ALT and AST in serum were detected ( $n=5 / g r o u p)$. (F, G) The levels of ALT and AST in mice liver were detected $(n=3 / g r o u p)$. (H, I) Hematoxylin and eosin staining (magnification, $\times 400$, scale bar, $20 \mu \mathrm{m}$ ). The stained sections were scored using a four-point scale from 0 to 3 , with $0,1,2$, and 3 representing no damage, mild damage, moderate damage, and severe damage, respectively ( $n=6 /$ group). (J, K) TUNEL staining and quantitative results of the percentage of TUNEL-positive cells in the liver of different experimental groups (magnification, $\times 400$, scale bar, $20 \mu \mathrm{m}$ ). Images were analyzed using the image analysis program ImageJ ( $n=6 /$ group). (L-N) Western blot assay was performed to detect the levels of Bcl-2 and Bax. The intensity of the bands was calculated using ImageJ software. $\beta$-actin served as the internal control $\left(n=3 /\right.$ group). ${ }^{*} p<0.05 ;{ }^{* \star} p<0.01 ;{ }^{\star \star \star} p<0.001$. n.s., not statistically significant. 

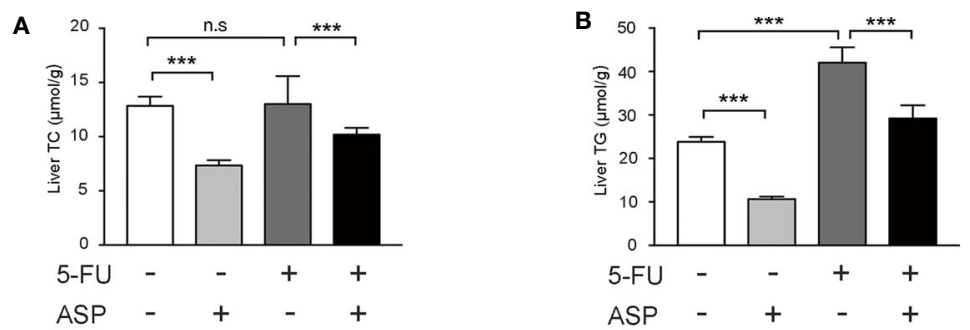

C

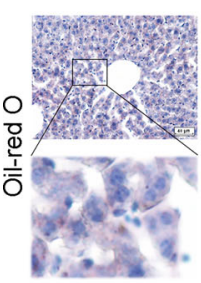

5-FU -

ASP -

E

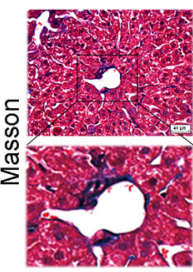

5 -FU -

ASP -

G

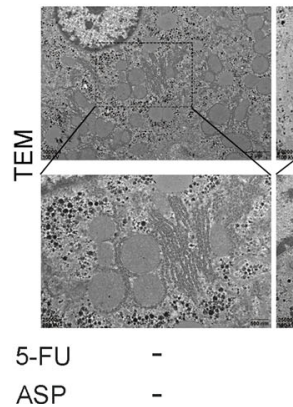

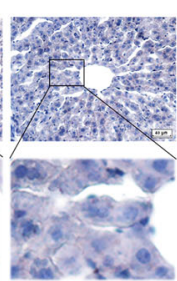

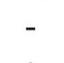

$+$

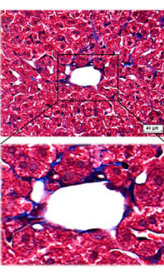

$-$

$+$

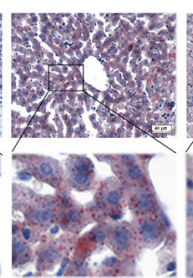

$+$

$-$

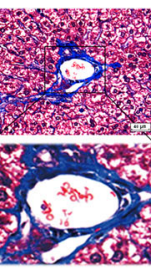

$+$

-
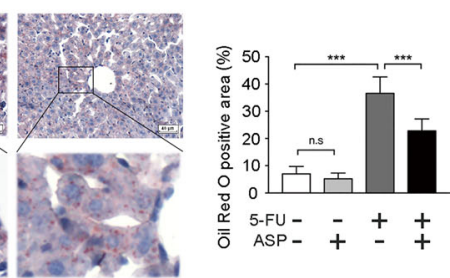

$\mathbf{F}$

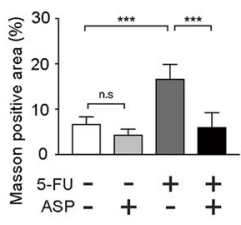

$+$

$+$
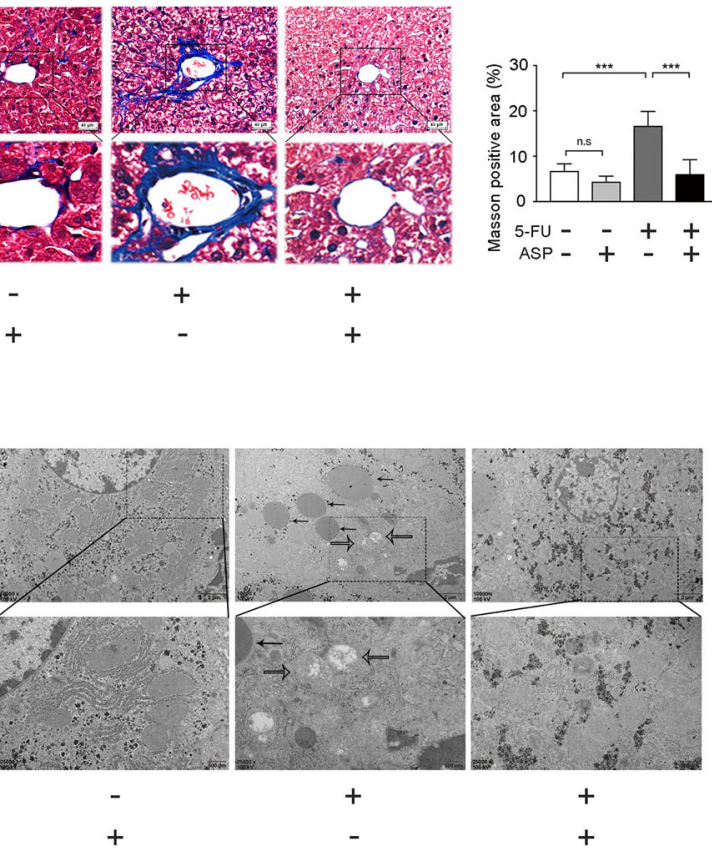

FIGURE 2 | ASP attenuated 5-FU-induced liver lipid deposition in vivo. (A) The level of TC was measured. (B) ASP treatment reversed the 5-FU-induced abnormal elevation of liver biochemical parameters and TG ( $n=6 /$ group). (C, D) Oil Red O staining (nuclei were stained purple, and lipids were stained red). ASP rescued the lipogenesis induced by 5-FU treatment (magnification, $\times 200$, scale bar, $40 \mu \mathrm{m}$ ). Images were analyzed using ImageJ ( $n=6 /$ group). (E, F) Masson staining of liver tissues (magnification, $\times 200$, scale bar, $40 \mu \mathrm{m})$. Images were analyzed using ImageJ ( $n=6 / g r o u p)$ (G) TEM micrograph of the mice liver. The solid arrow points to the lipid droplet, and the open arrow points to the swollen mitochondria (magnification, $\times 10000$ or $\times 25000$, scale bar, $2 \mu \mathrm{m}$ or $500 \mathrm{~nm}$, respectively). ${ }^{\star \star \star} \mathrm{p}<0.001$. n.s., not statistically significant.

microscopy, which showed that there was a large area of lipid deposition and mitochondrial swelling in the 5-FU group. Nevertheless, treatment with ASP alleviated lipid deposition and mitochondrial swelling (Figure 2G). These results suggested that ASP attenuates 5-FU-induced liver lipid deposition and mitochondrial swelling

\section{Effects of 5-FU Treatment on Lipid Synthesis, Lipid Uptake and Lipid- Metabolism-Related Gene Expression}

The expression of 18 genes related to lipid synthesis, uptake and metabolism was detected by RT-qPCR (Figures 3A, B). The expression of 3 genes in the 5-FU treatment group increased, and 
A

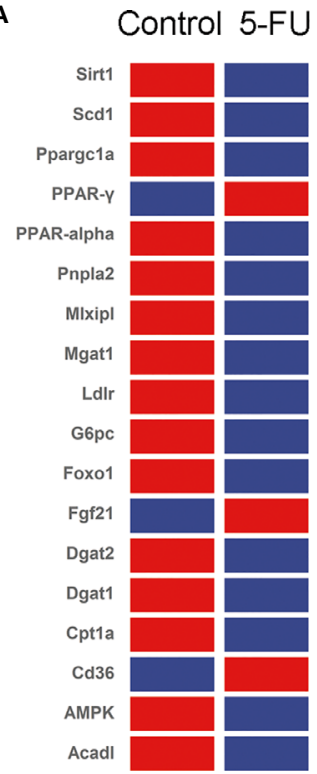

B

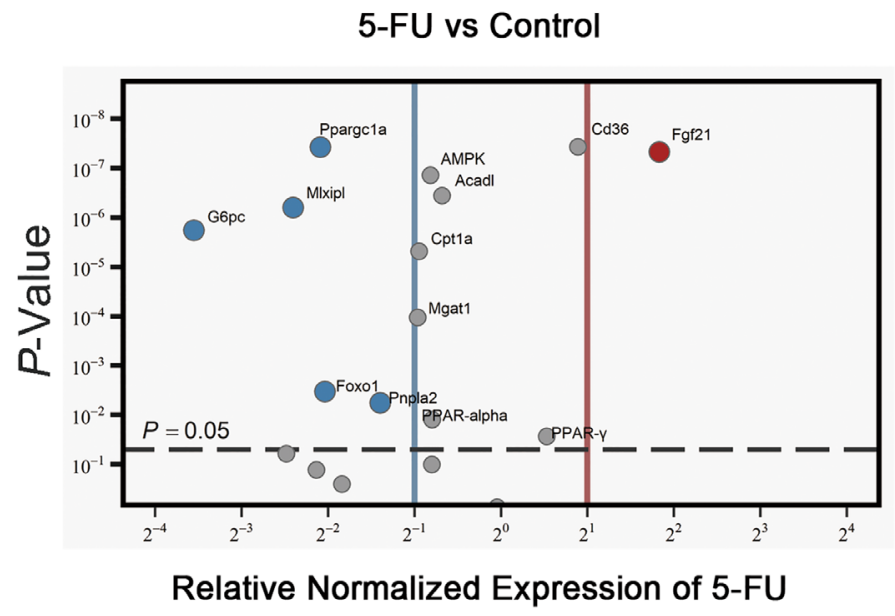

FIGURE 3 | Effects of 5-FU treatment on lipid synthesis, lipid uptake, and lipid metabolism-related gene expression. (A) The expression of genes in the livers of mice was detected by RT-qPCR assay $(n \geqq 6)$. Clustergram (Red means high mRNA expression level, and blue means low mRNA expression level). (B) Volcano $\geqq$ plot (the left side of the solid blue line represents genes whose mRNA expression level has been reduced by more than two times, the right side of the solid red line represents genes whose mRNA expression level has increased more than twice, and the genes above the gray dotted line indicate that the difference is statistically significant, $p<0.05$.

the expression of 15 genes decreased. The mRNA levels of Sirt1, Scd1, Dgat1, Dgat2, and Ldlr were not significantly different. The expression of PPAR- $\gamma$, CD36, and Fgf2 1 was increased significantly, while that of Ppargcla, Mlxipl, G6pc, Foxo1, Pnpla2, AMPK, Acadl, Cptla, Mgat1, and PPAR-alpha was decreased. Among them, PPAR-alpha and Cptla are essential genes that regulate fatty acid $\beta$-oxidation. Ppargcla is also a peroxisome proliferator-activated receptor coactivator. Pnpla2 is related to fatty acid hydrolysis. The results suggested that 5-FUinduced certain obstacles in the synthesis, uptake, and metabolism of lipids in the liver of mice, which may be related to the diminishment of fatty acid oxidation and hydrolysis in the liver.

\section{ASP Treatment Alleviated 5-FU-Induced Oxidative Damage in Mice}

We further detected the oxidative and antioxidant balance in each group. The results demonstrated that ASP treatment alleviated the increase in ROS levels induced by 5-FU (Figures 4A, B). In addition, the activities of CAT, GSH and SOD were detected. Also, the contents of MDA and NO were detected. ASP rescued the increase in MDA and NO content caused by 5 -FU (Figures 4C, D) and recovered the decreased levels of SOD, GSH and CAT caused by 5-FU in the livers of mice (Figures $\mathbf{4 E}-\mathbf{G}$ ). The results indicated that ASP treatment could reduce 5-FU-induced liver oxidative damage in mice.

\section{ASP Rescued the Inhibitory Effect of 5-FU on Nrf2 Pathway In Vivo}

The immunohistochemistry results showed that ASP treatment could relatively inhibit the increase in Keap1 expression induced by 5-FU (Figures 5A, B), which was further confirmed by western blot experiments (Figures 5I, J). Immunofluorescence results showed that 5 -FU treatment hindered the nuclear translocation of $\mathrm{Nrf2}$, and ASP treatment significantly enhanced the transcriptional activity of Nrf2; these results were further confirmed by western blot experiments (Figures 5C, D, G, H). The immunofluorescence results also showed that 5-FU treatment inhibited the relative expression of HO-1, and ASP treatment significantly promoted the expression of $\mathrm{HO}-1$; these results were further confirmed by western blot experiments (Figures 5E, F, I, J). Overall, these results indicated that 5-FU enhanced oxidative stress by inhibiting the activity of $\mathrm{Nrf} 2$ and that ASP rescued the inhibitory effect of $5-\mathrm{FU}$ on the Nrf2 pathway in vivo.

\section{ASP Treatment Reduced 5-FU-Induced Cell Oxidative Damage, the Inhibition of Lipid Metabolism Gene Expression and Cell Apoptosis In Vitro}

Mitochondria are the primary source of ROS, and excessive production of ROS can lead to cell damage. The smooth endoplasmic reticulum is related to lipid metabolism. The fatty 

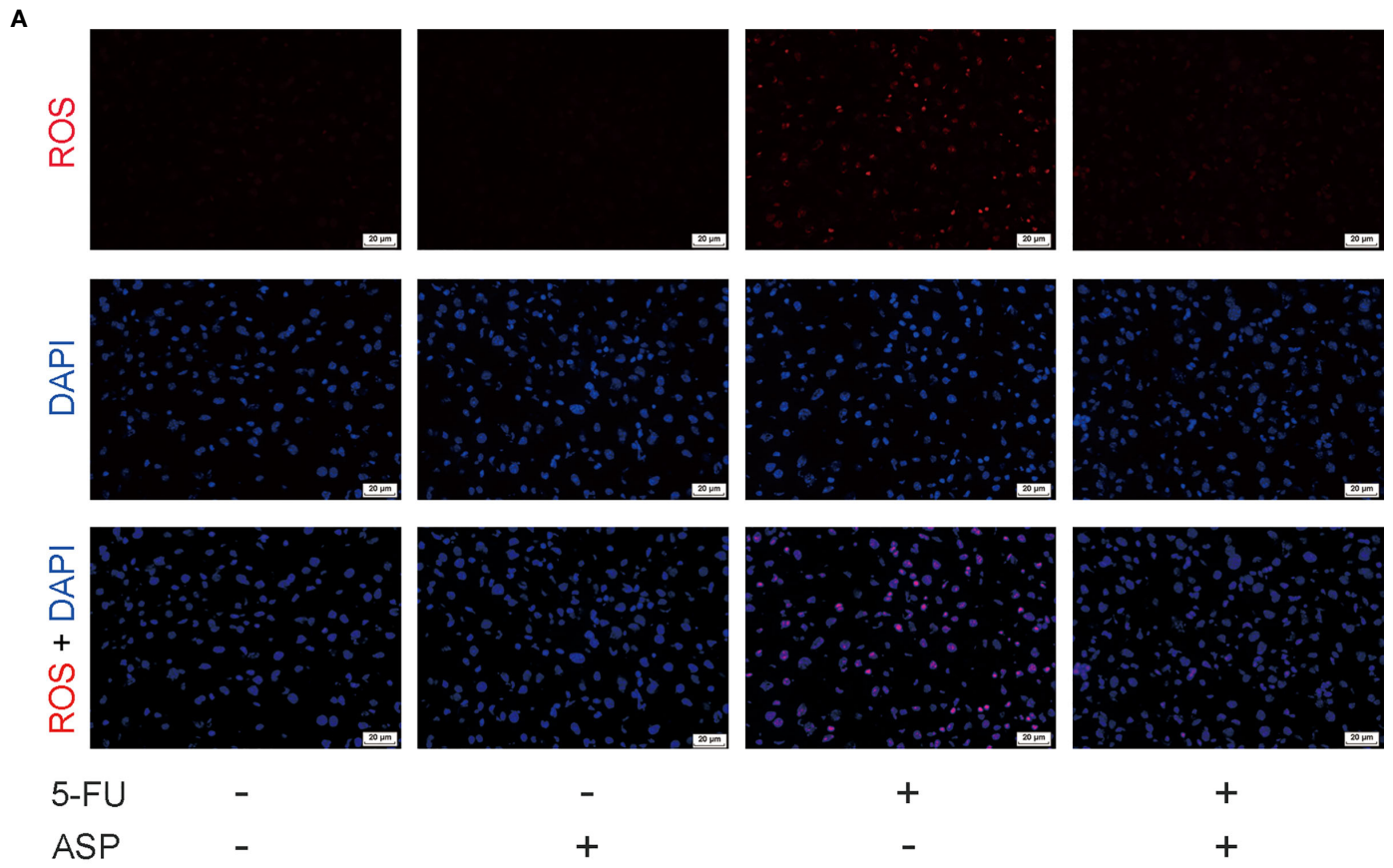

B

C

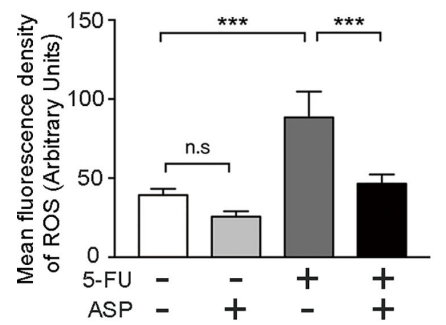

E

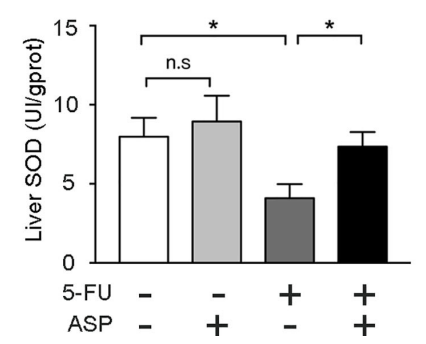

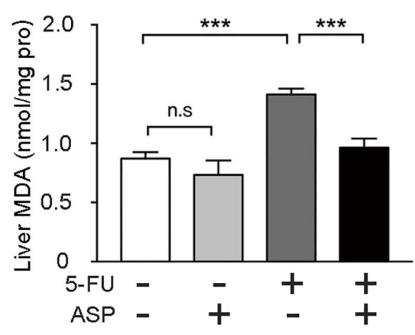

$\mathbf{F}$

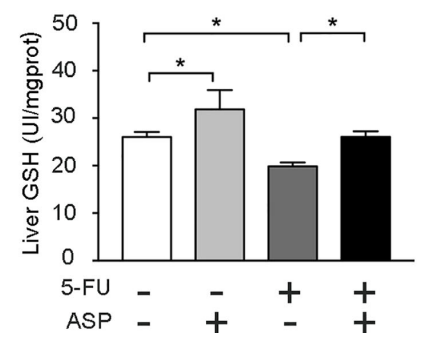

D

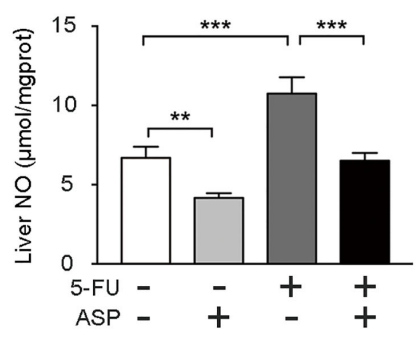

G

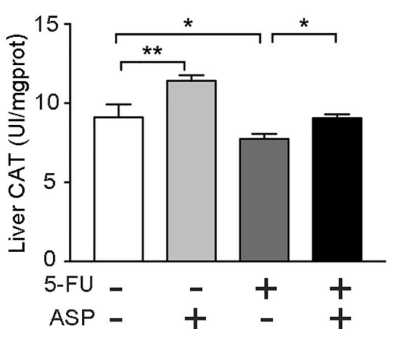

FIGURE 4 | ASP treatment relieved 5-FU-induced oxidative injury in mice. (A) The level of ROS was determined (magnification, $\times 400$, scale bar, 20 Hm). (B) The intensity of fluorescence was quantified under microscopy and analyzed using the image analysis program Image ( $n=6 / g r o u p)$. (C, D) ASP rescued the increased MDA and NO content caused by 5-FU in mice liver ( $n=3 /$ group). (E-G) ASP rescued the decreased levels of SOD, GSH, and CAT caused by 5 -FU in mice liver ( $n=3$ /group). ${ }^{*} p<0.05 ;{ }^{\star \star} P<0.01 ;{ }^{* \star *} p<0.001$. n.s., not statistically significant.

acids taken up by cells are decomposed by oxidoreductases in the smooth endoplasmic reticulum. Transmission electron microscopy results showed that a large amount of mitochondrial swelling and deformation was observed in the cytoplasm of the 5 -FU $(60 \mu \mathrm{g} / \mathrm{mL})$ treatment group, the expansion of smooth endoplasmic reticulum was also observed, and there was a large area of lipid deposition. ASP $(100 \mu \mathrm{g} / \mathrm{mL})$ treatment reversed the effect of $5-\mathrm{FU}$ on mitochondria, smooth 


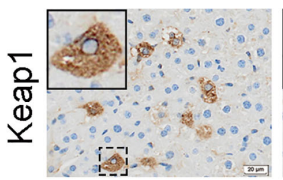

$$
5-\mathrm{FU}
$$
ASP

C
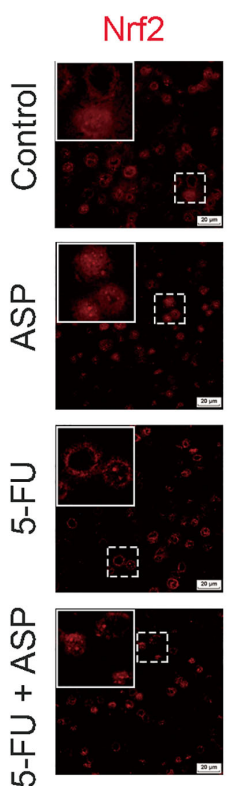

DAPI Nrf2 + DAPI
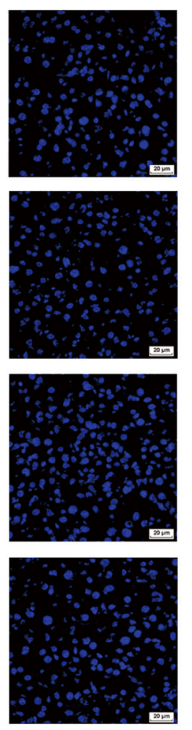
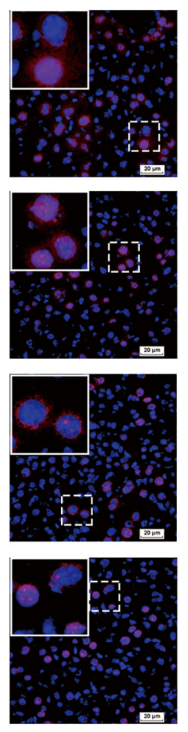

E

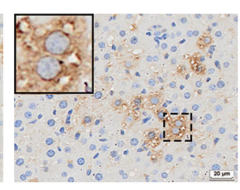

$+$

$+$
B

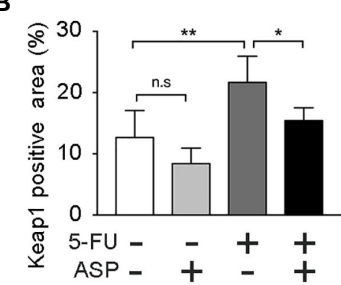

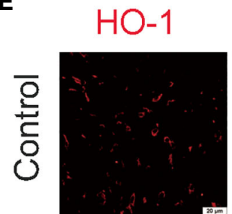

DAPI HO-1 + DAPI
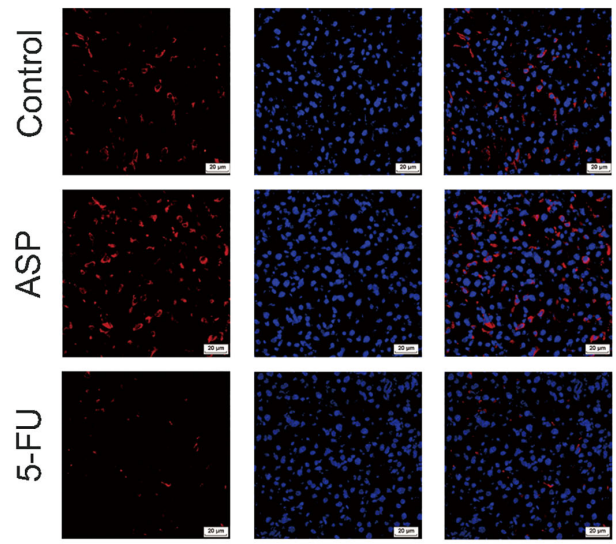

\begin{tabular}{l}
$\frac{0}{6}$ \\
+ \\
+ \\
$\square$ \\
\hline 1
\end{tabular}
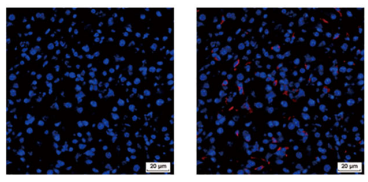

D

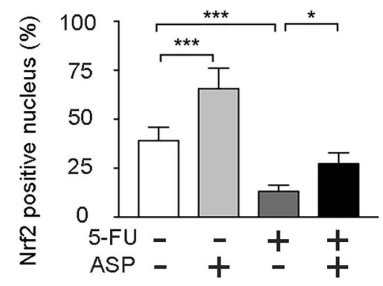

$\mathbf{F}$
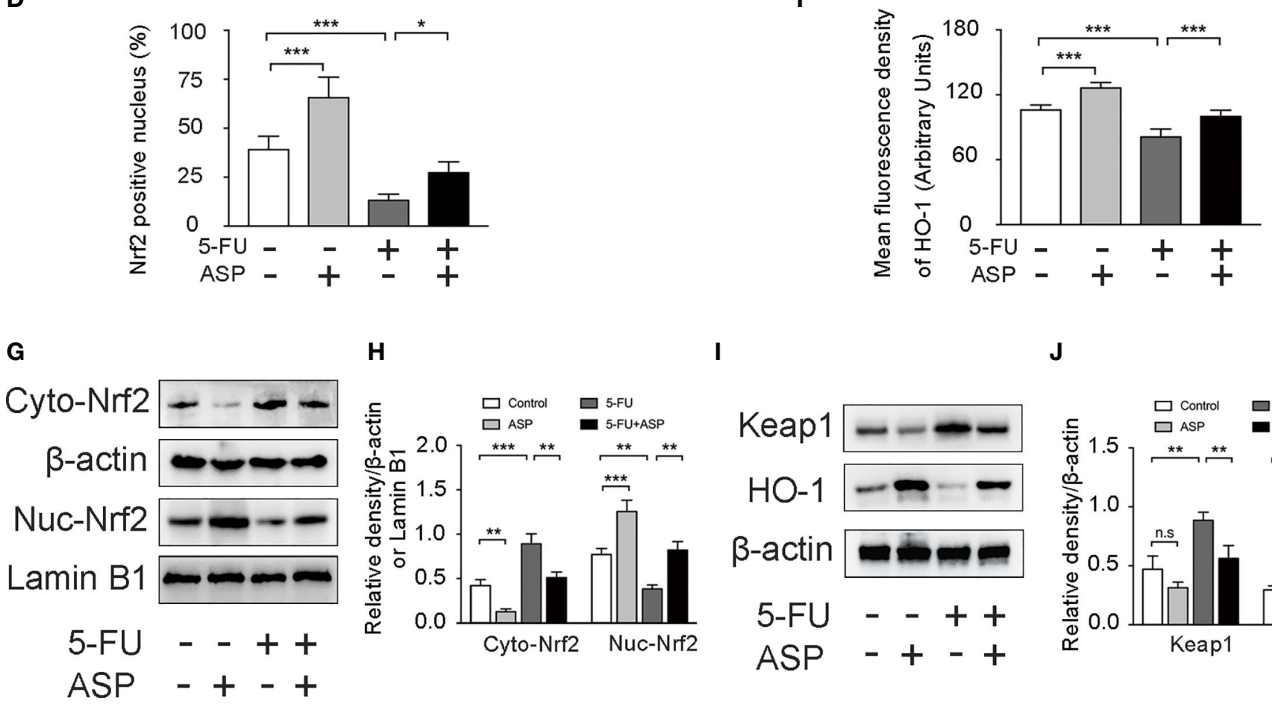

H

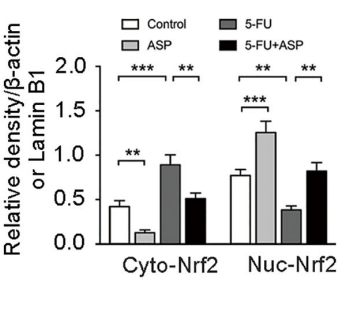

I

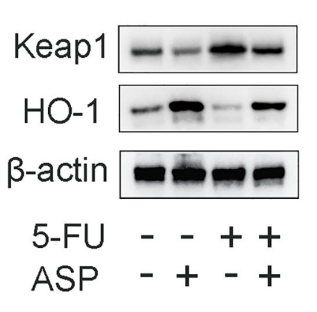

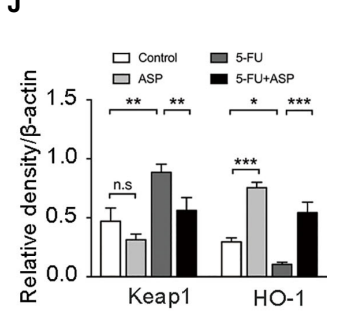

FIGURE 5 | ASP rescued the inhibition of the Keap1- Nrf2 pathway caused by 5-FU in vivo. (A) Immunohistochemistry was performed to detect the expression of Keap1 (magnification, $\times$ 400, scale bar, $20 \mu \mathrm{m}$ ). (B) A semiquantitative analysis of the ratio of Keap1-positive staining to the total field. (C) The nuclear translocation of Nrf2 was detected by immunofluorescence (IF) method, with Nrf2 antibody (red) and DAPI staining of the nucleus (blue) (magnification, $\times 400$, scale bar, $20 \mu \mathrm{m})$. (D) Quantitative results of the percentage of Nrf2-positive nuclei in the livers. (E) The immunofluorescence results showed that ASP alleviated the inhibition of HO-1 activity caused by 5-FU in the livers of mice (magnification, $\times$ 400, scale bar, $20 \mu \mathrm{m}$ ). (F) The mean fluorescence intensity of HO-1 was quantified ( $n=6$ /group). (G-J) Western blot assay was used to detect the cytoplasmic and nuclear levels of Nrf2 and the protein expression levels of Keap1 and HO-1in mouse livers ( $n=3 /$ group). The intensity of the bands was calculated using ImageJ software. $\beta$-actin and Lamin B1 (nuclear fraction) served as the internal control. ${ }^{*} p<0.05 ;{ }^{* \star} p<0.01 ;{ }^{* *} p<0.001$. n.s., not statistically significant. 
A

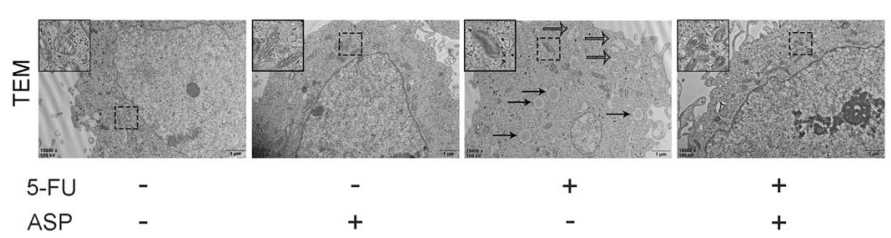

$\square$ Control $\square$ ASP $\square$ 5-FU $\square$ 5-FU+ASP
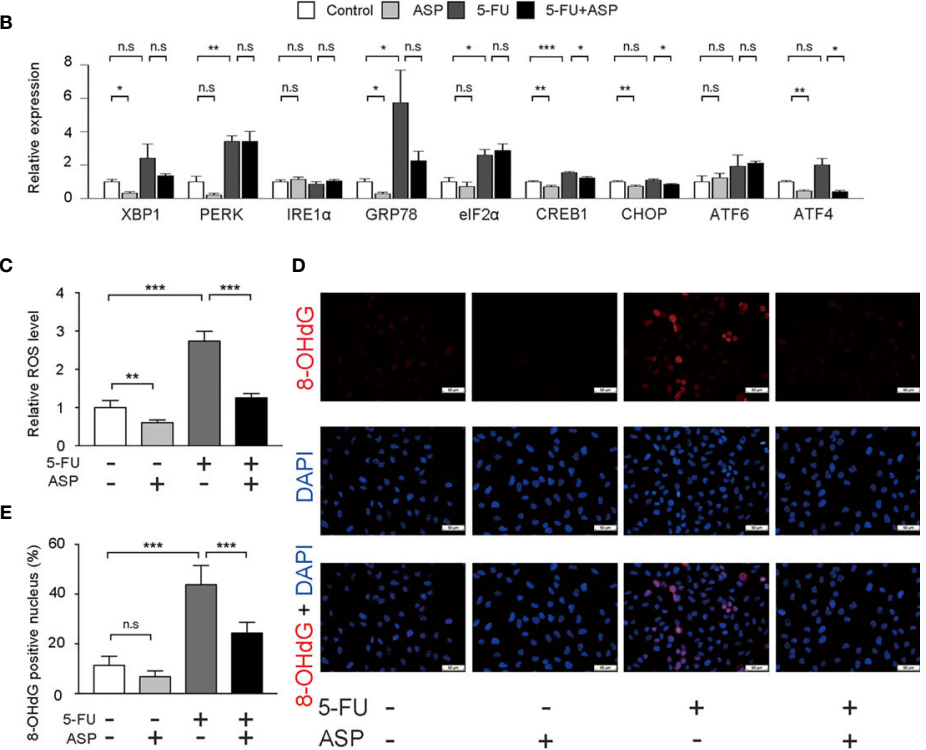

$\mathbf{F}$
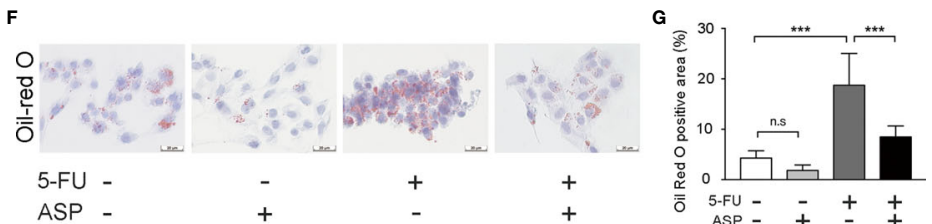

$\square$ Control $\square$ ASP $\square$ 5-FU $\square$ 5-FU+ASP
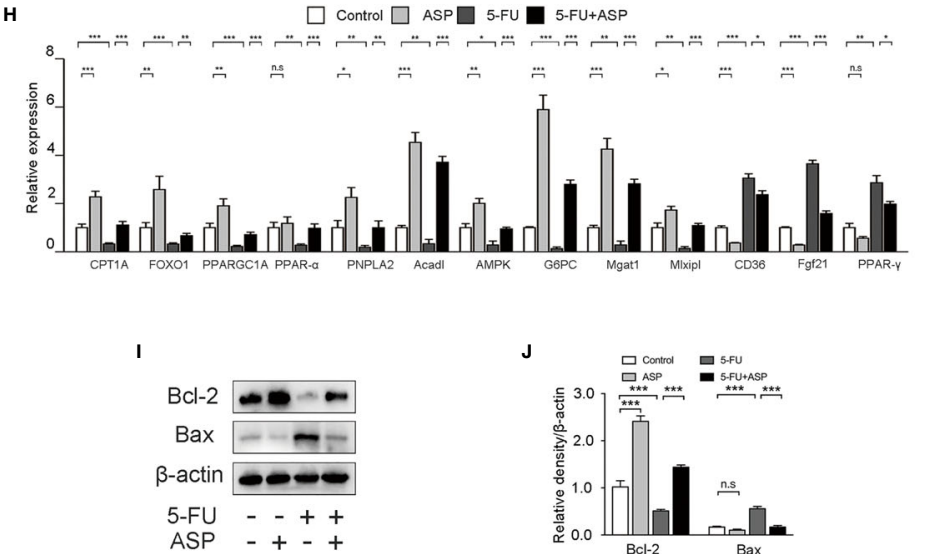

FIGURE 6 | ASP treatment reduced 5-FU-induced cell oxidative damage and apoptosis and inhibited lipid metabolism gene expression in vitro. (A) Cell ultrastructure was observed under a TEM (magnification, $\times 15000$, scale bar, $1 \mu \mathrm{m}$ ). The solid arrow points to the lipid droplet, and the open arrow points to the swollen endoplasmic reticulum. The swollen mitochondria are shown within the box at a higher magnification. (B) The expression of 9 genes related to endoplasmic reticulum stress was detected by RT-qPCR. $\beta$-actin was probed as an internal control $(n=3)$. (C) ROS production of the indicated groups ( $n=6 /$ group). (D, E) Representative immunofluorescence images and the quantification of 8-OHdG staining in cells (magnification, $\times 400$, scale bar, $20 \mu \mathrm{m}$ ). (F) Oil Red O staining of cells (magnification, $\times$ 400, scale bar, $20 \mu \mathrm{m})$. (G) Oil Red O-positive areas were analyzed using ImageJ ( $n=6 / \mathrm{group})$. (H) The expression of lipid synthesis and metabolism-related genes in cells was detected by RT-qPCR assay $(n=3)$. $\beta$-actin was probed as an internal control. (I) Western blot assay was performed to determine the expression levels of Bax and Bcl-2. (J) The intensity of the bands was calculated using ImageJ software $\left(n=3 /\right.$ group). ${ }^{*} p<0.05 ;{ }^{* \star} p<0.01 ;{ }^{* \star *} p<0.001$. n.s., not statistically significant. 
endoplasmic reticulum and lipid deposition to a certain extent (Figure 6A). The expression of 9 genes related to endoplasmic reticulum stress was detected by RT-qPCR (Figure 6B). Compared with the control group, the expression of 8 genes in the 5-FU treatment group increased, and the expression of 1 gene decreased. The mRNA levels of XBP1, IRE1 $\alpha$, CHOP, ATF6, and ATF4 were not significantly different between the two groups. The expression of PERK, GRP78, eIF2 $\alpha$ and CREB1 was increased significantly. RT-qPCR results demonstrated that ASP treatment could restore the increase in the expression of the 5-FU-induced endoplasmic reticulum stress-related genes CREB1, CHOP and ATF4 (Figure 6B). The ROS content in the 5 -FU group was increased as compared with the control group, and ASP treatment partially eliminated intracellular ROS (Figure 6C). 8-OHdG is one of the major products of DNA oxidation. ASP treatment alleviated the 5-FU-induced increase in 8-OHdG levels in MIHA cells (Figures 6D, E). In MIHA cells, the Oil Red O-positive area increased significantly after 5-FU treatment for $24 \mathrm{~h}$, and intracellular lipid droplets increased. After treatment with ASP, the changes were alleviated (Figures 6F, G), suggesting that ASP has an improvement effect on 5-FU-induced lipid deposition in MIHA cells. RTqPCR results demonstrated that ASP treatment could significantly restore the decrease in the expression of the 5-FUinduced lipid synthesis and metabolism-related genes CPT1A, FOXO1, PPARGC1A, PPAR-alpha, PNPLA2, Acadl, AMPK, G6PC, Mgat1 and Mlxipl. And ASP treatment could significantly inhibit the increase in the expression of the 5-FUinduced CD36, Fgf2 1 and PPAR- $\gamma($ Figure 6H). In addition, the results indicated that the pro-apoptotic protein Bax was increased in the 5-FU group. In contrast, the anti-apoptotic protein Bcl-2 was significantly reduced; this situation was reversed after ASP treatment (Figures 6I, J), which indicated that 5-FU was inhibited by ASP treatment-induced hepatocyte apoptosis, which was consistent with the in vivo data. The above results suggest that ASP treatment can reduce 5-FU-induced cell oxidative damage and inhibit lipid metabolism gene expression and cell apoptosis in vitro.

\section{ASP Rescued the Inhibitory Effect of 5-FU on Nrf2 Pathway In Vitro}

The immunofluorescence results in MIHA cells showed that 5FU treatment hindered the nuclear translocation of Nrf2, and ASP treatment significantly enhanced the transcriptional activity of Nrf2; these were further confirmed by western blot experiments (Figures 7A, B, E, F). The immunofluorescence results also showed that 5 -FU treatment reduced the expression of HO-1, whereas ASP treatment enhanced the expression of HO-1; these results were further confirmed by western blot experiments (Figures 7C, D, G, H). The results demonstrated that ASP treatment reduced the increase in Keap1 expression induced by 5 -FU (Figures 7G, H). Overall, these results indicated that 5-FU enhanced oxidative stress by inhibiting Nrf2 activity, and ASP rescued the inhibitory effect of 5-FU on the Nrf2 pathway in vitro.

\section{DISCUSSION}

Chemotherapy, as one of the most commonly used measures for treating malignant tumors, is widely used in the clinical treatment of tumors. Tumor therapeutic doses of chemotherapy drugs kill tumor cells while also damaging normal cells, resulting in damage to normal cells and organs (30). It has been reported that the chemotherapy drugs oxaliplatin, asparaginase, carmustine, mercaptopurine, and methotrexate can be hepatotoxicity. The mechanism of hepatotoxicity may be that chemotherapy drugs induce inflammation, liver cell apoptosis, oxidative stress damage, and abnormal liver metabolism (31-34). The chemotherapy drug 5fluorouracil is widely used clinically to treat various tumors, including colorectal cancer, breast cancer, and liver cancer. However, liver toxicity caused by chemotherapy often prevents the administration of sufficient effective drug doses to patients (4). Studies have shown that 5-FU treatment induces portal fibrosis and increased apoptosis, and at the ultrastructural level, vesicular rough endoplasmic reticulum and atrophic mitochondria are observed (7). Our previous studies have proven that the chemotherapy drug 5 -FU can cause oxidative damage to bone marrow stromal cells and changes in the secretion of biologically active substances. The mechanism of 5 -FU injury to the liver is still unclear and remains to be further explored and studied (2). Our results proved that ASP could reduce 5-FU-induced liver oxidative damage and watery degeneration, lipid accumulation, and vacuolation. As a result, the mitochondria of liver cells and the endoplasmic reticulum swell, thereby delaying cell apoptosis and reducing hepatotoxicity. ASP treatment significantly promotes nuclear translocation of liver Nrf2 protein, reduces liver oxidative stress levels, and reverses 5-FU-mediated liver pathological changes in vivo and in vitro.

Damages such as oxidants, $\gamma$-ray radiation and chemotherapy can put cells in oxidative stress, leading to abnormal metabolism and the apoptosis of cells $(35,36)$. In this study, 5 -FU induced a loss of liver weight and increased TG, AST, and ALT levels, suggesting decreased liver function and triglyceride deposition in mice. Through various histomorphological staining techniques, severe lipid deposition and a large number of hepatocytes, swelling, necrosis, vacuolation and fibrosis were observed in the livers of the 5-FU group. Our study showed many fatty acid deposits and abnormal mitochondria in the 5-FU group in vivo and in vitro. In this experiment, 5-FU treatment caused damage to normal liver cells. However, the mechanism is not precise.

Many experiments have shown that oxidative stress can lead to endoplasmic reticulum stress, which induces apoptosis (37-39). Endoplasmic reticulum is an essential place for lipid synthesis. Abnormal endoplasmic reticulum function can cause disorders of lipid anabolism (40-42). In this study, by observing the ultrastructure of liver cells, we observed that after 5-FU treatment, the endoplasmic reticulum structure in the liver cells was abnormal, mainly manifested as swelling. RT-qPCR was used to detect the expression of 9 endoplasmic reticulum stress-related genes, 

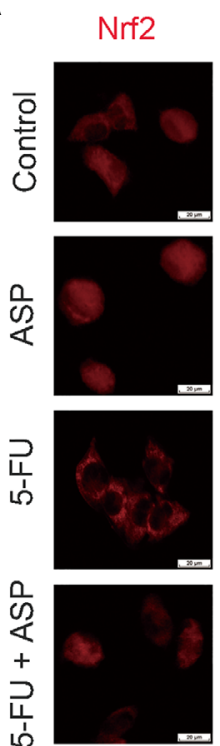

B

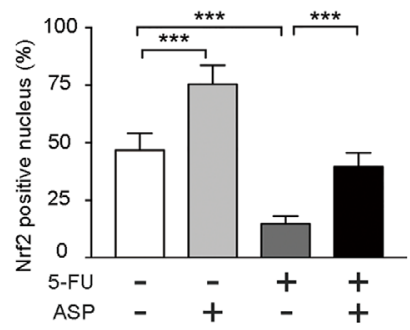

E

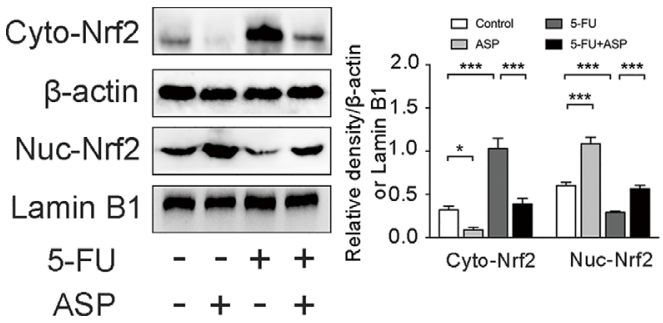

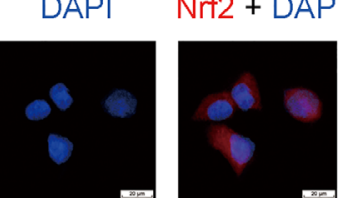
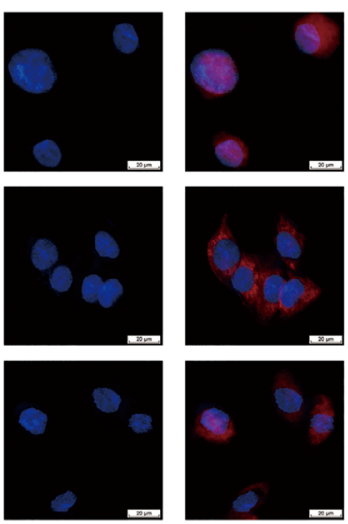

D
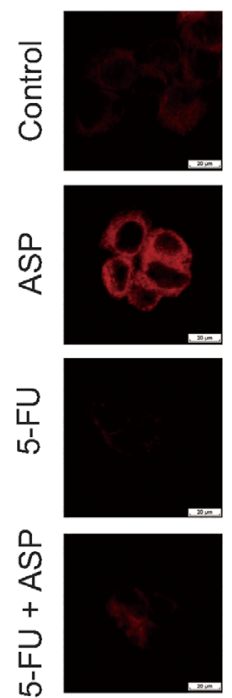

$\mathrm{HO}-1$ + DAPI
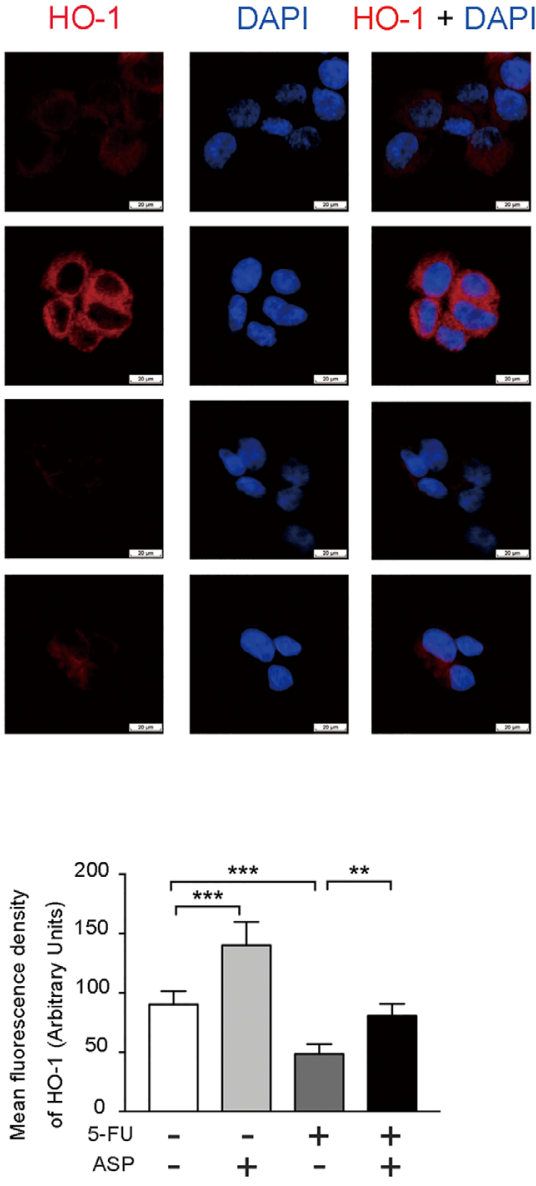

G

H
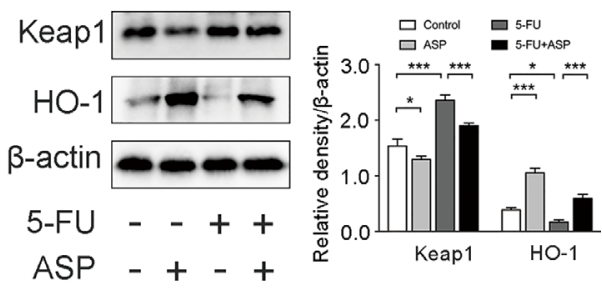

FIGURE 7 | ASP rescued the inhibition of the Keap1- Nrf2 pathway caused by 5-FU in vitro. (A) The nuclear translocation of Nrf2 was detected by immunofluorescence (IF) method, with Nrf2 antibody (red) and DAPI staining of the nucleus (blue) (magnification, $\times 400$, scale bar, $20 \mu \mathrm{m}$ ). (B) Quantitative results of the percentage of Nrf2-positive nuclei in the cells. (C) The immunofluorescence results showed that ASP alleviated the inhibition of HO-1 activity caused by 5-FU in cells (magnification, $\times 400$, scale bar, $20 \mu \mathrm{m}$ ). (D) The mean fluorescence intensity of HO-1 was quantified ( $n=6 / \mathrm{group})$. (E-H) Western blot assay was used to detect the cytoplasmic and nuclear levels of Nrf2 and the protein expression levels of Keap1 and HO- 1 in the cells ( $n=3 / g r o u p)$. The intensity of the bands was calculated using ImageJ software. $\beta$-actin and Lamin B1 (nuclear fraction) served as the internal control. ${ }^{*} p<0.05 ;{ }^{* \star} p<0.01 ;{ }^{* \star *} p<0.001$.

including XBP1, PERK, IRE1 $\alpha$, GRP78, eIF2 $\alpha$, CREB1, CHOP, ATF6, and ATF4. The expression of 8 genes in the 5-FU treatment group increased, and the expression of 1 gene decreased compared with the control group. The mRNA levels of XBP1, IRE1 $\alpha$, CHOP, ATF6, and ATF4 were not significantly different between the two groups. The expression of PERK, GRP78, eIF2 $\alpha$, and CREB1 was increased significantly. RT-qPCR results also demonstrated that ASP treatment could restore the increase in the expression of the 5FU-induced endoplasmic reticulum stress-related genes CREB1, CHOP, and ATF4. These results suggest that ASP can alleviate 
the endoplasmic reticulum stress caused by 5 -FU. In addition, RTqPCR was measured to detect the expression of 18 lipid synthesis-, uptake- and metabolism-related genes in mouse livers. The expression changes of 13 genes related to synthesis and metabolism in the 5-FU group were significantly different from the control group. This suggests that endoplasmic reticulum stress can indeed cause disorders of lipid synthesis and metabolism. Among them, PPAR-alpha can metabolize NEFAs that the liver has taken up, thereby protecting the liver from lipid peroxidation damage (43). PPAR-alpha is a nuclear receptor activated by a ligand. PPAR-alpha is the main lipid oxidation factor in the liver. PPARalpha regulates target genes involved in $\beta$ oxidation, such as Cptla. PPAR-alpha promotes lipid oxidation in liver cells, thereby reducing lipids in animal models. Metabolism and steatosis are beneficial (44, 45). Ppargcla is a crucial physiological transcription regulator of oxidative metabolism and mitochondrial biogenesis (46). Ppargcla promotes mitochondrial biogenesis, oxidation capacity and fatty acid $\beta$ oxidation by increasing the expression and activation of various transcription factors (such as PPAR-alpha) (47). In terms of fatty acid decomposition, the key TG hydrolase in the liver is Pnpla2, and Sirt1 can regulate fat mobilization through Foxo1mediated Pnpla2 expression (48-50). Therefore, we focused on verifying the expression of five lipid-metabolism-related genes in vitro, and the results obtained were consistent with those of the in vivo experiments. After 5-FU treatment, the expression of 5 genes related to lipid metabolism decreased significantly. It has been suggested that 5 -FU can induce oxidative stress to cause abnormal endoplasmic reticulum function to affect lipid synthesis, inhibit the expression of lipid metabolism genes, and ultimately lead to lipid deposition. Studies have also demonstrated that lipid deposition aggravates the production of ROS, which leads to cell apoptosis (51-53). In our experiment, we also observed that after 5FU treatment, the apoptotic cells in the mouse liver increased. Western blot results further demonstrated the upregulation of Bax and the downregulation of $\mathrm{Bcl}-2$ in the 5-FU group both in vivo and in vitro. However, there are few drugs target antiapoptotic proteins for clinical treatment. The occurrence of apoptosis is closely related to oxidative stress. Theoretically, eliminating oxidative stress can help alleviate apoptosis. Therefore, finding an effective, low-toxicity natural medicine that can exert antioxidant effects is necessary.

Under normal circumstances, the ROS content remains in balance $(54,55)$. Excessive ROS content will cause oxidative stress, leading to inflammation, apoptosis, and other disease processes $(56,57)$. Additionally, free fatty acids can cause lipotoxicity by disrupting the balance of the reactive oxygen system (58). Excessive production of ROS can cause lipid peroxidation and a disturbance of antioxidant and peroxidase activity to cause cell dysfunction and cell apoptosis $(59,60)$. Since the mitochondria can generate most of the ROS, mitochondria are considered the main contributors to oxidative stress $(61,62)$. In this study, 5-FU induced an increase in ROS levels both in vivo and in vitro. Furthermore, the ultrastructure of the liver and hepatocytes was observed. The liver mitochondria treated with 5FU were swollen, and most of the mitochondria in the liver cells were abnormal. We speculate that 5-FU can cause a large amount of ROS production by damaging mitochondria morphology and causing dysfunction. The enzyme activities of SOD, GSH and CAT are essential indicators to assess the capacity to resist oxidation. In this study, 5-FU-induced a decrease in the enzyme activity of SOD, GSH, and CAT, suggesting that 5-FU caused damage to the liver's antioxidant capacity. MDA is an end product of lipid peroxidation, a good marker of oxidative damage. NO is a highly reactive free radical in biology. In this study, 5-FU-induced an increase in the levels of MDA and NO, suggesting that 5-FU aggravates oxidative stress in the liver. In addition, the level of ROS in cells rises uncontrollably, which can induce the accumulation of DNA damage to promote the apoptosis process (63-65). Oxidative stress triggers DNA damage, leading to the DNA damage response (DDR). 8OHdG is one of the major products of DNA oxidation (66, 67). Our results indicated that after 5-FU treatment of hepatocytes, the expression of $8-\mathrm{OHdG}$ was significantly increased, suggesting that 5-FU-induced oxidative stress caused DDR. Our results show that 5-FU can damage the mitochondrial function and increase ROS production, which in turn reduces the activity of antioxidant enzymes and increases peroxides content, leading to DNA damage and DDR, thereby promoting cell apoptosis.

Nrf2 is a crucial regulator in the cellular oxidative stress response (68), regulated by Keap1 to regulate the expression of antioxidant proteins (69). It has been found that the Nrf2 signaling pathway is one of the core pathways of the cellular antioxidant response, which can significantly induce the body's endogenous antioxidant response (70). The abnormal Nrf2 signaling pathway will aggravate oxidative stress damage and destroy the normal redox balance in the cell $(71,72)$. Keap 1 protein is sensitive to oxidative stress. Under normal conditions, Keap1 and Nrf2 exist as dimers in the cytoplasm. Once exposed to oxidative stress, the two dissociate and Nrf2 is transcribed to regulate the transcriptional activation of a series of cytoprotective genes (73), such as heme oxygenase-1 (HO-1) (74). HO-1 has anti-apoptosis, anti-inflammatory, and antioxidant functions $(75,76)$. The effect of 5 -FU on the Nrf2 pathway and its mechanism remains to be explored. We detected the expression of Nrf2 pathway proteins in mouse livers and MIHA cells. The results showed that in both mice and cells treated with 5 FU, the level of Keap 1 was relatively increased. In contrast, the level of HO-1 was decreased, which was further confirmed by immunohistochemistry experiments and immunofluorescence experiments. The results of immunofluorescence and western blot experiments showed that after 5-FU induction, the level of Nrf2 in the nucleus was significantly reduced, and the transport of Nrf2 into the nucleus was reduced. It is suggested that 5-FU induction can inhibit Nrf2 nuclear translocation, thereby reducing the level of the downstream antioxidant protein $\mathrm{HO}-1$, leading to further weakening of the antioxidant ability of cells and intensifying oxidative stress.

ASP is extracted from the root of Dang Gui. The main components of ASP include galactonic acid, glucuronic acid, galactose, arabinose, xylose, and rhamnose $(77,78)$. It has been proven that ASP has beneficial effects in many disease models, such as diabetes, osteoarthritis, and anemia (79-81). Our previous studies have shown that ASP slows down the senescence of bone marrow 
stromal cells and hematopoietic cells by inhibiting oxidative stress $(2,25)$. A study showed that angelica polysaccharides could reduce CCl4-induced liver fibrosis (82). However, to date, the effect of ASP on 5-FU-induced liver hepatotoxicity and the mechanism by which it occurs is still unknown.

This study demonstrated that ASP protects the liver from structural and functional damage caused by 5 -FU, mainly by reducing the symptoms of apoptosis. ASP reversed the nuclear translocation disorder of $\mathrm{Nrf} 2$ caused by $5-\mathrm{FU}$, leading to increased expression of antioxidant genes to inhibit oxidative stress, restoring the function of the mitochondria and endoplasmic reticulum to reduce lipid deposition and DNA damage, and alleviating apoptosis. This study provides not only a protective agent for liver injury caused by the chemotherapy drug 5-FU but also a novel target for treating the liver injury.

\section{DATA AVAILABILITY STATEMENT}

The original contributions presented in the study are included in the article/Supplementary Material. Further inquiries can be directed to the corresponding author.

\section{ETHICS STATEMENT}

The animal study was reviewed and approved by Chongqing Medical University Animal Care and Use Committee.

\section{REFERENCES}

1. Longley DB, Harkin DP, Johnston PG. 5-Fluorouracil: Mechanisms of Action and Clinical Strategies. Nat Rev Cancer (2003) 3:330-8. doi: 10.1038/nrc1074

2. Xiao H, Xiong L, Song X, Jin P, Chen L, Chen X, et al. Angelica Sinensis Polysaccharides Ameliorate Stress-Induced Premature Senescence of Hematopoietic Cell via Protecting Bone Marrow Stromal Cells From Oxidative Injuries Caused by 5-Fluorouracil. Int J Mol Sci (2017) 18:2265. doi: $10.3390 /$ ijms 18112265

3. Chibber S, Farhan M, Hassan I, Naseem I. White Light-Mediated Cu (II)-5FU Interaction Augments the Chemotherapeutic Potential of 5-FU: An In Vitro study. Tumour Biol (2011) 32:881-92. doi: 10.1007/s13277-011-0189-y

4. Coronado-Cerda EE, Franco-Molina MA, Mendoza-Gamboa E, PradoGarcía H, Rivera-Morales LG, Zapata-Benavides P, et al. In Vivo Chemoprotective Activity of Bovine Dialyzable Leukocyte Extract in Mouse Bone Marrow Cells Against Damage Induced by 5-Fluorouracil. J Immunol Res (2016) 2016:6942321. doi: 10.1155/2016/6942321

5. Lamberti M, Porto S, Marra M, Zappavigna S, Grimaldi A, Feola D, et al. 5Fluorouracil Induces Apoptosis in Rat Cardiocytes Through Intracellular Oxidative Stress. J Exp Clin Cancer Res (2012) 31:60. doi: 10.1186/1756-9966-31-60

6. Rashid S, Ali N, Nafees S, Hasan SK, Sultana S. Mitigation of 5-Fluorouracil Induced Renal Toxicity by Chrysin via Targeting Oxidative Stress and Apoptosis in Wistar Rats. Food Chem Toxicol (2014) 66:185-93. doi: $10.1016 /$ j.fct.2014.01.026

7. El-Sayyad HI, Ismail MF, Shalaby FM, Abou-El-Magd RF, Gaur RL, Fernando A, et al. Histopathological Effects of Cisplatin, Doxorubicin and 5-Flurouracil (5-FU) on the Liver of Male Albino Rats. Int J Biol Sci (2009) 5:466-73. doi: $10.7150 /$ ijbs.5.466

8. Malhi H, Gores GJ. Cellular and Molecular Mechanisms of Liver Injury. Gastroenterology (2008) 134:1641-54. doi: 10.1053/j.gastro.2008.03.002

\section{AUTHOR CONTRIBUTIONS}

DZ, YW, LW, and JL designed the study. DZ, HX, ZW, GL, and YC performed the experiments. $\mathrm{HL}, \mathrm{JH}$, and DL analyzed the results. DZ, YW, and JL wrote the manuscript. All authors contributed to the article and approved the submitted version.

\section{FUNDING}

This study was supported by the National Natural Science Foundation of China (Nos. 81873103, 81673748).

\section{ACKNOWLEDGMENTS}

We would like to thank the resources and services provided by the School of Life Sciences, Chongqing Medical University.

\section{SUPPLEMENTARY MATERIAL}

The Supplementary Material for this article can be found online at: https://www.frontiersin.org/articles/10.3389/fonc.2021. 720620/full\#supplementary-material

9. Spahis S, Delvin E, Borys JM, Levy E. Oxidative Stress as a Critical Factor in Nonalcoholic Fatty Liver Disease Pathogenesis. Antioxid Redox Signal (2017) 26:519-41. doi: 10.1089/ars.2016.6776

10. Chen X, Xue H, Fang W, Chen K, Chen S, Yang W, et al. Adropin Protects Against Liver Injury in Nonalcoholic Steatohepatitis via the Nrf2 Mediated Antioxidant Capacity. Redox Biol (2019) 21:101068. doi: 10.1016/ j.redox.2018.101068

11. Liu Y, Wen PH, Zhang XX, Dai Y, He Q. Breviscapine Ameliorates Ccl4 -Induced Liver Injury in Mice Through Inhibiting Inflammatory Apoptotic Response and ROS Generation. Int J Mol Med (2018) 42:755-68. doi: 10.3892/ ijmm.2018.3651

12. Choudhury S, Ghosh S, Mukherjee S, Gupta P, Bhattacharya S, Adhikary A, et al. Pomegranate Protects Against Arsenic-Induced P53-Dependent ROSMediated Inflammation and Apoptosis in Liver Cells. J Nutr Biochem (2016) 38:25-40. doi: 10.1016/j.jnutbio.2016.09.001

13. Yu Y, Sun X, Gu J, Yu C, Wen Y, Gao Y, et al. Deficiency of DJ-1 Ameliorates Liver Fibrosis Through Inhibition of Hepatic ROS Production and Inflammation. Int J Biol Sci (2016) 12:1225-35. doi: 10.7150/ijbs.15154

14. Maher J, Yamamoto M. The Rise of Antioxidant Signaling-the Evolution and Hormetic Actions of Nrf2. Toxicol Appl Pharmacol (2010) 244:4-15. doi: 10.1016/j.taap.2010.01.011

15. Kobayashi A, Kang MI, Watai Y, Tong KI, Shibata T, Uchida K, et al. Oxidative and Electrophilic Stresses Activate Nrf2 Through Inhibition of Ubiquitination Activity of Keap1. Mol Cell Biol (2006) 26:221-9. doi: 10.1128/ mcb.26.1.221-229.2006

16. Itoh K, Tong KI, Yamamoto M. Molecular Mechanism Activating Nrf2-Keap1 Pathway in Regulation of Adaptive Response to Electrophiles. Free Radic Biol Med (2004) 36:1208-13. doi: 10.1016/j.freeradbiomed.2004.02.075

17. Son Y, Lee JH, Chung HT, Pae HO. Therapeutic Roles of Heme Oxygenase-1 in Metabolic Diseases: Curcumin and Resveratrol Analogues as Possible 
Inducers of Heme Oxygenase-1. Oxid Med Cell Longev (2013) 2013:639541. doi: $10.1155 / 2013 / 639541$

18. Yang Y, Liu L, Zhang X, Jiang X, Wang L. Tanshinone IIA Prevents Rifampicin-Induced Liver Injury by Regulating BSEP/NTCP Expression via Epigenetic Activation of NRF2. Liver Int (2020) 40:141-54. doi: 10.1111/ liv. 14262

19. Chen Y, Liu K, Zhang J, Hai Y, Wang P, Wang H, et al. C-Jun NH(2) -Terminal Protein Kinase Phosphorylates the Nrf2-ECH Homology 6 Domain of Nuclear Factor Erythroid 2-Related Factor 2 and Downregulates Cytoprotective Genes in Acetaminophen-Induced Liver Injury in Mice. Hepatology (2020) 71:1787-801. doi: 10.1002/hep.31116

20. Lyu H, Wang H, Li L, Zhu J, Chen F, Chen Y, et al. Hepatocyte-Specific Deficiency of Nrf2 Exacerbates Carbon Tetrachloride-Induced Liver Fibrosis via Aggravated Hepatocyte Injury and Subsequent Inflammatory and Fibrogenic Responses. Free Radic Biol Med (2020) 150:136-47. doi: 10.1016/ j.freeradbiomed.2020.02.015

21. Chao WW, Lin BF. Bioactivities of Major Constituents Isolated From Angelica Sinensis (Danggui). Chin Med (2011) 6:29. doi: 10.1186/17498546-6-29

22. Chen Y, Duan JA, Qian D, Guo J, Song B, Yang M. Assessment and Comparison of Immunoregulatory Activity of Four Hydrosoluble Fractions of Angelica Sinensis In Vitro on the Peritoneal Macrophages in ICR Mice. Int Immunopharmacol (2010) 10:422-30. doi: 10.1016/j.intimp.2010.01.004

23. Bunel V, Antoine MH, Nortier J, Duez P, Stévigny C. Potential Nephroprotective Effects of the Chinese Herb Angelica Sinensis Against Cisplatin Tubulotoxicity. Pharm Biol (2015) 53:985-94. doi: 10.3109/ 13880209.2014.951726

24. Bradley RR, Cunniff PJ, Pereira BJ, Jaber BL. Hematopoietic Effect of Radix Angelicae Sinensis in a Hemodialysis Patient. Am J Kidney Dis (1999) 34:34954. doi: 10.1016/s0272-6386(99)70367-7

25. Mu X, Zhang Y, Li J, Xia J, Chen X, Jing P, et al. Angelica Sinensis Polysaccharide Prevents Hematopoietic Stem Cells Senescence in DGalactose-Induced Aging Mouse Model. Stem Cells Int (2017) 2017:3508907. doi: 10.1155/2017/3508907

26. Zeng D, Zhou P, Jiang R, Li XP, Huang SY, Li DY, et al. Evodiamine Inhibits Vasculogenic Mimicry in HCT116 Cells by Suppressing Hypoxia-Inducible Factor 1-Alpha-Mediated Angiogenesis. Anticancer Drugs (2021) 32:314-22. doi: $10.1097 / \mathrm{cad} .0000000000001030$

27. Hu L, Tian K, Zhang T, Fan CH, Zhou P, Zeng D, et al. Cyanate Induces Oxidative Stress Injury and Abnormal Lipid Metabolism in Liver Through Nrf2/HO-1. Molecules (2019) 24:3231. doi: 10.3390/molecules24183231

28. Qi R, Jiang R, Xiao H, Wang Z, He S, Wang L, et al. Ginsenoside Rg1 Protects Against D-Galactose Induced Fatty Liver Disease in a Mouse Model via FOXO1 Transcriptional Factor. Life Sci (2020) 254:117776. doi: 10.1016/ j.lfs.2020.117776

29. Peng X, Yang Y, Tang L, Wan J, Dai J, Li L, et al. Therapeutic Benefits of Apocynin in Mice With Lipopolysaccharide/D-Galactosamine-Induced Acute Liver Injury via Suppression of the Late Stage Pro-Apoptotic AMPK/JNK Pathway. BioMed Pharmacother (2020) 125:110020. doi: 10.1016/j.biopha. 2020.110020

30. Banfi A, Bianchi G, Galotto M, Cancedda R, Quarto R. Bone Marrow Stromal Damage After Chemo/Radiotherapy: Occurrence, Consequences and Possibilities of Treatment. Leuk Lymphoma (2001) 42:863-70. doi: 10.3109/ 10428190109097705

31. Tabassum H, Waseem M, Parvez S, Qureshi MI. Oxaliplatin-Induced Oxidative Stress Provokes Toxicity in Isolated Rat Liver Mitochondria. Arch Med Res (2015) 46:597-603. doi: 10.1016/j.arcmed.2015.10.002

32. Kamal N, Koh C, Samala N, Fontana RJ, Stolz A, Durazo F, et al. Asparaginase-Induced Hepatotoxicity: Rapid Development of Cholestasis and Hepatic Steatosis. Hepatol Int (2019) 13:641-48. doi: 10.1007/s12072019-09971-2

33. Ramadori G, Cameron S. Effects of Systemic Chemotherapy on the Liver. Ann Hepatol (2010) 9:133-43. doi: 10.1016/S1665-2681(19)31651-5

34. Famurewa AC, Ufebe OG, Egedigwe CA, Nwankwo OE, Obaje GS. Virgin Coconut Oil Supplementation Attenuates Acute Chemotherapy Hepatotoxicity Induced by Anticancer Drug Methotrexate via Inhibition of Oxidative Stress in Rats. BioMed Pharmacother (2017) 87:437-42. doi: 10.1016/j.biopha.2016.12.123
35. Salminen A, Ojala J, Kaarniranta K. Apoptosis and Aging: Increased Resistance to Apoptosis Enhances the Aging Process. Cell Mol Life Sci (2011) 68:1021-31. doi: 10.1007/s00018-010-0597-y

36. Childs BG, Baker DJ, Kirkland JL, Campisi J, van Deursen JM. Senescence and Apoptosis: Dueling or Complementary Cell Fates? EMBO Rep (2014) 15:1139-53. doi: 10.15252/embr.201439245

37. Kunitomi C, Harada M, Takahashi N, Azhary JMK, Kusamoto A, Nose E, et al. Activation of Endoplasmic Reticulum Stress Mediates Oxidative StressInduced Apoptosis of Granulosa Cells in Ovaries Affected by Endometrioma. Mol Hum Reprod (2020) 26:40-52. doi: 10.1093/molehr/gaz066

38. Wu Y, Reece EA, Zhong J, Dong D, Shen WB, Harman CR, et al. Type 2 Diabetes Mellitus Induces Congenital Heart Defects in Murine Embryos by Increasing Oxidative Stress, Endoplasmic Reticulum Stress, and Apoptosis. Am J Obstet Gynecol (2016) 215:e1-66.e10:366. doi: 10.1016/j.ajog. 2016.03.036

39. Guo JJ, Ma LL, Shi HT, Zhu JB, Wu J, Ding ZW, et al. Alginate Oligosaccharide Prevents Acute Doxorubicin Cardiotoxicity by Suppressing Oxidative Stress and Endoplasmic Reticulum-Mediated Apoptosis. Mar Drugs (2016) 14:231. doi: 10.3390/md14120231

40. Jacquemyn J, Cascalho A, Goodchild RE. The Ins and Outs of Endoplasmic Reticulum-Controlled Lipid Biosynthesis. EMBO Rep (2017) 18:1905-21. doi: $10.15252 / \mathrm{embr} .201643426$

41. Kwiatek JM, Han GS, Carman GM. Phosphatidate-Mediated Regulation of Lipid Synthesis at the Nuclear/Endoplasmic Reticulum Membrane. Biochim Biophys Acta Mol Cell Biol Lipids (2020) 1865:158434. doi: 10.1016/ j.bbalip.2019.03.006

42. Zhang L, Wang HH. The Essential Functions of Endoplasmic Reticulum Chaperones in Hepatic Lipid Metabolism. Dig Liver Dis (2016) 48:709-16. doi: 10.1016/j.dld.2016.03.016

43. Pawlak M, Lefebvre P, Staels B. Molecular Mechanism of Pparo Action and its Impact on Lipid Metabolism, Inflammation and Fibrosis in Non-Alcoholic Fatty Liver Disease. J Hepatol (2015) 62:720-33. doi: 10.1016/j.jhep. 2014.10.039

44. Zhang H, Yang L, Wang Y, Huang W, Li Y, Chen S, et al. Oxymatrine Alleviated Hepatic Lipid Metabolism via Regulating MiR-182 in NonAlcoholic Fatty Liver Disease. Life Sci (2020) 257:118090. doi: 10.1016/ j.lfs. 2020.118090

45. Xie K, He X, Chen K, Sakao K, Hou DX. Ameliorative Effects and Molecular Mechanisms of Vine Tea on Western Diet-Induced NAFLD. Food Funct (2020) 11:5976-91. doi: 10.1039/d0fo00795a

46. Zhang Q, Ma XF, Dong MZ, Tan J, Zhang J, Zhuang LK, et al. MiR-30b-5p Regulates the Lipid Metabolism by Targeting PPARGC1A in Huh-7 Cell Line. Lipids Health Dis (2020) 19:76. doi: 10.1186/s12944-020-01261-3

47. Tan HWS, Anjum B, Shen HM, Ghosh S, Yen PM, Sinha RA. Lysosomal Inhibition Attenuates Peroxisomal Gene Transcription via Suppression of PPARA and PPARGC1A Levels. Autophagy (2019) 15:1455-59. doi: 10.1080/ 15548627.2019.1609847

48. Zhang X, Zhang CC, Yang H, Soni KG, Wang SP, Mitchell GA, et al. An Epistatic Interaction Between Pnpla2 and Lipe Reveals New Pathways of Adipose Tissue Lipolysis. Cells (2019) 8:395. doi: 10.3390/cells8050395

49. Filali-Mouncef Y, Hunter C, Roccio F, Zagkou S, Dupont N, Primard C, et al. The Ménage À Trois of Autophagy, Lipid Droplets and Liver Disease. Autophagy (2021) 2:1-24. doi: 10.1080/15548627.2021.1895658

50. Chen G, Yu D, Nian X, Liu J, Koenig RJ, Xu B, et al. LncRNA SRA Promotes Hepatic Steatosis Through Repressing the Expression of Adipose Triglyceride Lipase (ATGL). Sci Rep (2016) 6:35531. doi: 10.1038/srep35531

51. Wang Y, Xue J, Li Y, Zhou X, Qiao S, Han D. Telmisartan Protects Against High Glucose/High Lipid-Induced Apoptosis and Insulin Secretion by Reducing the Oxidative and ER Stress. Cell Biochem Funct (2019) 37:16168. doi: 10.1002/cbf. 3383

52. Wójcik P, Žarković N, Gęgotek A, Skrzydlewska E. Involvement of Metabolic Lipid Mediators in the Regulation of Apoptosis. Biomolecules (2020) 10:402. doi: 10.3390/biom10030402

53. Flores-Romero H, Ros U, García-Sáez AJ. A Lipid Perspective on Regulated Cell Death. Int Rev Cell Mol Biol (2020) 351:197-236. doi: 10.1016/ bs.ircmb.2019.11.004

54. Galvan DL, Badal SS, Long J, Chang BH, Schumacker PT, Overbeek PA, et al. Real-Time In Vivo Mitochondrial Redox Assessment Confirms Enhanced 
Mitochondrial Reactive Oxygen Species in Diabetic Nephropathy. Kidney Int (2017) 92:1282-87. doi: 10.1016/j.kint.2017.05.015

55. Kirkman DL, Muth BJ, Ramick MG, Townsend RR, Edwards DG. Role of Mitochondria-Derived Reactive Oxygen Species in Microvascular Dysfunction in Chronic Kidney Disease. Am J Physiol Renal Physiol (2018) 314:F423-f29. doi: 10.1152/ajprenal.00321.2017

56. Alfaradhi MZ, Fernandez-Twinn DS, Martin-Gronert MS, Musial B, Fowden A, Ozanne SE. Oxidative Stress and Altered Lipid Homeostasis in the Programming of Offspring Fatty Liver by Maternal Obesity. Am J Physiol Regul Integr Comp Physiol (2014) 307:R26-34. doi: 10.1152/ajpregu. 00049.2014

57. Scheuer H, Gwinner W, Hohbach J, Gröne EF, Brandes RP, Malle E, et al. Oxidant Stress in Hyperlipidemia-Induced Renal Damage. Am J Physiol Renal Physiol (2000) 278:F63-74. doi: 10.1152/ajprenal.2000.278.1.F63

58. Egnatchik RA, Leamy AK, Noguchi Y, Shiota M, Young JD. PalmitateInduced Activation of Mitochondrial Metabolism Promotes Oxidative Stress and Apoptosis in H4IIEC3 Rat Hepatocytes. Metabolism (2014) 63:283-95. doi: 10.1016/j.metabol.2013.10.009

59. Mihalas BP, De Iuliis GN, Redgrove KA, McLaughlin EA, Nixon B. The Lipid Peroxidation Product 4-Hydroxynonenal Contributes to Oxidative StressMediated Deterioration of the Ageing Oocyte. Sci Rep (2017) 7:6247. doi: 10.1038/s41598-017-06372-z

60. Damgaard D, Bjørn ME, Jensen PØ, Nielsen CH. Reactive Oxygen Species Inhibit Catalytic Activity of Peptidylarginine Deiminase. J Enzyme Inhib Med Chem (2017) 32:1203-8. doi: 10.1080/14756366.2017.1368505

61. Fulle S, Protasi F, Di Tano G, Pietrangelo T, Beltramin A, Boncompagni S, et al. The Contribution of Reactive Oxygen Species to Sarcopenia and Muscle Ageing. Exp Gerontol (2004) 39:17-24. doi: 10.1016/j.exger.2003.09.012

62. Brookes PS. Mitochondrial H(+) Leak and ROS Generation: An Odd Couple. Free Radic Biol Med (2005) 38:12-23. doi: 10.1016/j.freeradbiomed.2004.10.016

63. Garinis GA, van der Horst GT, Vijg J, Hoeijmakers JH. DNA Damage and Ageing: New-Age Ideas for an Age-Old Problem. Nat Cell Biol (2008) 10:1241-7. doi: 10.1038/ncb1108-1241

64. Barzilai A, Yamamoto K. DNA Damage Responses to Oxidative Stress. DNA Repair (Amst) (2004) 3:1109-15. doi: 10.1016/j.dnarep.2004.03.002

65. Vilenchik MM, Knudson AG. Endogenous DNA Double-Strand Breaks: Production, Fidelity of Repair, and Induction of Cancer. Proc Natl Acad Sci USA (2003) 100:12871-6. doi: 10.1073/pnas.2135498100

66. Sperka T, Wang J, Rudolph KL. DNA Damage Checkpoints in Stem Cells, Ageing and Cancer. Nat Rev Mol Cell Biol (2012) 13:579-90. doi: 10.1038/nrm3420

67. Hensley K, Robinson KA, Gabbita SP, Salsman S, Floyd RA. Reactive Oxygen Species, Cell Signaling, and Cell Injury. Free Radic Biol Med (2000) 28:145662. doi: 10.1016/s0891-5849(00)00252-5

68. Tsai JJ, Dudakov JA, Takahashi K, Shieh JH, Velardi E, Holland AM, et al. Nrf2 Regulates Haematopoietic Stem Cell Function. Nat Cell Biol (2013) 15:309-16. doi: 10.1038/ncb2699

69. Magesh S, Chen Y, Hu L. Small Molecule Modulators of Keap1-Nrf2-are Pathway as Potential Preventive and Therapeutic Agents. Med Res Rev (2012) 32:687-726. doi: 10.1002/med.21257

70. Ma Q. Role of Nrf2 in Oxidative Stress and Toxicity. Annu Rev Pharmacol Toxicol (2013) 53:401-26. doi: 10.1146/annurev-pharmtox-011112-140320

71. Kubben N, Zhang W, Wang L, Voss TC, Yang J, Qu J, et al. Repression of the Antioxidant NRF2 Pathway in Premature Aging. Cell (2016) 165:1361-74. doi: $10.1016 /$ j.cell.2016.05.017

72. Li Y, Shi J, Sun X, Li Y, Duan Y, Yao H. Theaflavic Acid From Black Tea Protects PC12 Cells Against ROS-Mediated Mitochondrial Apoptosis Induced by OGD/R via Activating Nrf2/ARE Signaling Pathway. J Nat Med (2020) 74:238-46. doi: 10.1007/s11418-019-01333-4
73. Bollong MJ, Lee G, Coukos JS, Yun H, Zambaldo C, Chang JW, et al. A Metabolite-Derived Protein Modification Integrates Glycolysis With KEAP1NRF2 Signalling. Nature (2018) 562:600-4. doi: 10.1038/s41586-018-0622-0

74. Maruyama A, Mimura J, Itoh K. Non-Coding RNA Derived From the Region Adjacent to the Human HO-1 E2 Enhancer Selectively Regulates HO-1 Gene Induction by Modulating Pol II Binding. Nucleic Acids Res (2014) 42:13599614. doi: $10.1093 /$ nar/gku1169

75. Abd El-Twab SM, Hussein OE, Hozayen WG, Bin-Jumah M, Mahmoud AM. Chicoric Acid Prevents Methotrexate-Induced Kidney Injury by Suppressing NF-kb/NLRP3 Inflammasome Activation and Up-Regulating Nrf2/are/HO-1 Signaling. Inflammation Res (2019) 68:511-23. doi: 10.1007/s00011-01901241-z

76. Dang X, He B, Ning Q, Liu Y, Guo J, Niu G, et al. Alantolactone Suppresses Inflammation, Apoptosis and Oxidative Stress in Cigarette Smoke-Induced Human Bronchial Epithelial Cells Through Activation of Nrf2/HO-1 and Inhibition of the NF-kb Pathways. Respir Res (2020) 21:95. doi: 10.1186/ s12931-020-01358-4

77. Ma P, Sun C, Li W, Deng W, Adu-Frimpong M, Yu J, et al. Extraction and Structural Analysis of Angelica Sinensis Polysaccharide With Low Molecular Weight and Its Lipid-Lowering Effect on Nonalcoholic Fatty Liver Disease. Food Sci Nutr (2020) 8:3212-24. doi: 10.1002/fsn3.1581

78. Yi L, Liang Y, Wu H, Yuan D. The Analysis of Radix Angelicae Sinensis (Danggui). J Chromatogr A (2009) 1216:1991-2001. doi: 10.1016/ j.chroma.2008.07.033

79. Wang K, Cao P, Shui W, Yang Q, Tang Z, Zhang Y. Angelica Sinensis Polysaccharide Regulates Glucose and Lipid Metabolism Disorder in Prediabetic and Streptozotocin-Induced Diabetic Mice Through the Elevation of Glycogen Levels and Reduction of Inflammatory Factors. Food Funct (2015) 6:902-9. doi: 10.1039/c4fo00859f

80. Zhuang C, Wang Y, Zhang Y, Xu N. Oxidative Stress in Osteoarthritis and Antioxidant Effect of Polysaccharide From Angelica Sinensis. Int J Biol Macromol (2018) 115:281-86. doi: 10.1016/j.ijbiomac.2018.04.083

81. Li MM, Zhang Y, Wu J, Wang KP. Polysaccharide From Angelica Sinensis Suppresses Inflammation and Reverses Anemia in Complete Freund's Adjuvant-Induced Rats. Curr Med Sci (2020) 40:265-74. doi: 10.1007/ s11596-020-2183-3

82. Wang K, Wang J, Song M, Wang H, Xia N, Zhang Y. Angelica Sinensis Polysaccharide Attenuates Ccl(4)-Induced Liver Fibrosis via the IL-22/STAT3 Pathway. Int J Biol Macromol (2020) 162:273-83. doi: 10.1016/j.ijbiomac. 2020.06.166

Conflict of Interest: The authors declare that the research was conducted in the absence of any commercial or financial relationships that could be construed as a potential conflict of interest.

Publisher's Note: All claims expressed in this article are solely those of the authors and do not necessarily represent those of their affiliated organizations, or those of the publisher, the editors and the reviewers. Any product that may be evaluated in this article, or claim that may be made by its manufacturer, is not guaranteed or endorsed by the publisher.

Copyright $\odot 2021$ Zeng, Wang, Chen, Li, Li, Xiao, Hou, Wang, Hu, Wang and Li. This is an open-access article distributed under the terms of the Creative Commons Attribution License (CC BY). The use, distribution or reproduction in other forums is permitted, provided the original author(s) and the copyright owner(s) are credited and that the original publication in this journal is cited, in accordance with accepted academic practice. No use, distribution or reproduction is permitted which does not comply with these terms. 